\title{
Bio-composites for Fused Filament Fabrication. Effects of Maleic Anhydride Grafting on Poly(Lactic Acid) and Microcellulose
}

Daniele Rigotti ( $\boldsymbol{\sim}$ daniele.rigotti-1@unitn.it)

University of Trento: Universita degli Studi di Trento https://orcid.org/0000-0002-2337-8669

Luca Fambri

University of Trento: Universita degli Studi di Trento

Alessandro Pegoretti

University of Trento: Universita degli Studi di Trento

\section{Research Article}

Keywords: poly(lactic acid), microcellulose, fused filament fabrication, maleic anhydride

Posted Date: September 3rd, 2021

DOl: https://doi.org/10.21203/rs.3.rs-839482/v1

License: (c) (i) This work is licensed under a Creative Commons Attribution 4.0 International License.

Read Full License

Version of Record: A version of this preprint was published at Progress in Additive Manufacturing on January 24th, 2022. See the published version at https://doi.org/10.1007/s40964-022-00264-z. 


\title{
Bio-composites for fused filament fabrication. Effects of maleic anhydride grafting on poly(lactic acid) and microcellulose
}

\author{
Daniele Rigotti*, Luca Fambri and Alessandro Pegoretti. \\ University of Trento, Department of Industrial Engineering and INSTM Research Unit \\ Via Sommarive 938123 Trento (Italy) \\ daniele.rigotti-1@unitn.it \\ 0000-0002-2337-8669
}

\begin{abstract}
Composite filaments consisting of poly(lactic acid) (PLA) and micro crystalline cellulose (MCC) were successfully used for additive manufacturing (AM) by fused filament fabrication (FFF). PLA and MCC bio-composites were obtained by direct mixing in a melt compounder; maleic anhydride (MAH) was also grafted onto PLA in reactive mixing stage to evaluate its effect on the final properties of the printed material. Filaments with various concentrations of MCC (up to a maximum content of $10 \mathrm{wt} \%$ ) were produced with a single screw extruder and used to feed a commercial desktop FFF printer. Upon grafting of PLA with MAH, a more coherent interfacial morphology between PLA and MCC was detected by electron microscopy analysis. The thermal degradation of the PLA was unaffected by the presence of MCC and MAH. According to differential scanning calorimetry and dynamic mechanical analysis results, micro-cellulose acted as nucleating agent for PLA. In fact, the crystallization peak shifted towards lowers temperature and a synergistic effect when MCC was added to PLA grafted with MAH was observed possibly due to the increase of the chain mobility. Micro cellulose led to an increase in the stiffness of the material in both filaments and 3D printed specimen, however, a different fracture behavior was observed due to the peculiar structure of printed samples.
\end{abstract}

Keywords: poly(lactic acid), microcellulose, fused filament fabrication, maleic anhydride 


\section{Graphical abstract}

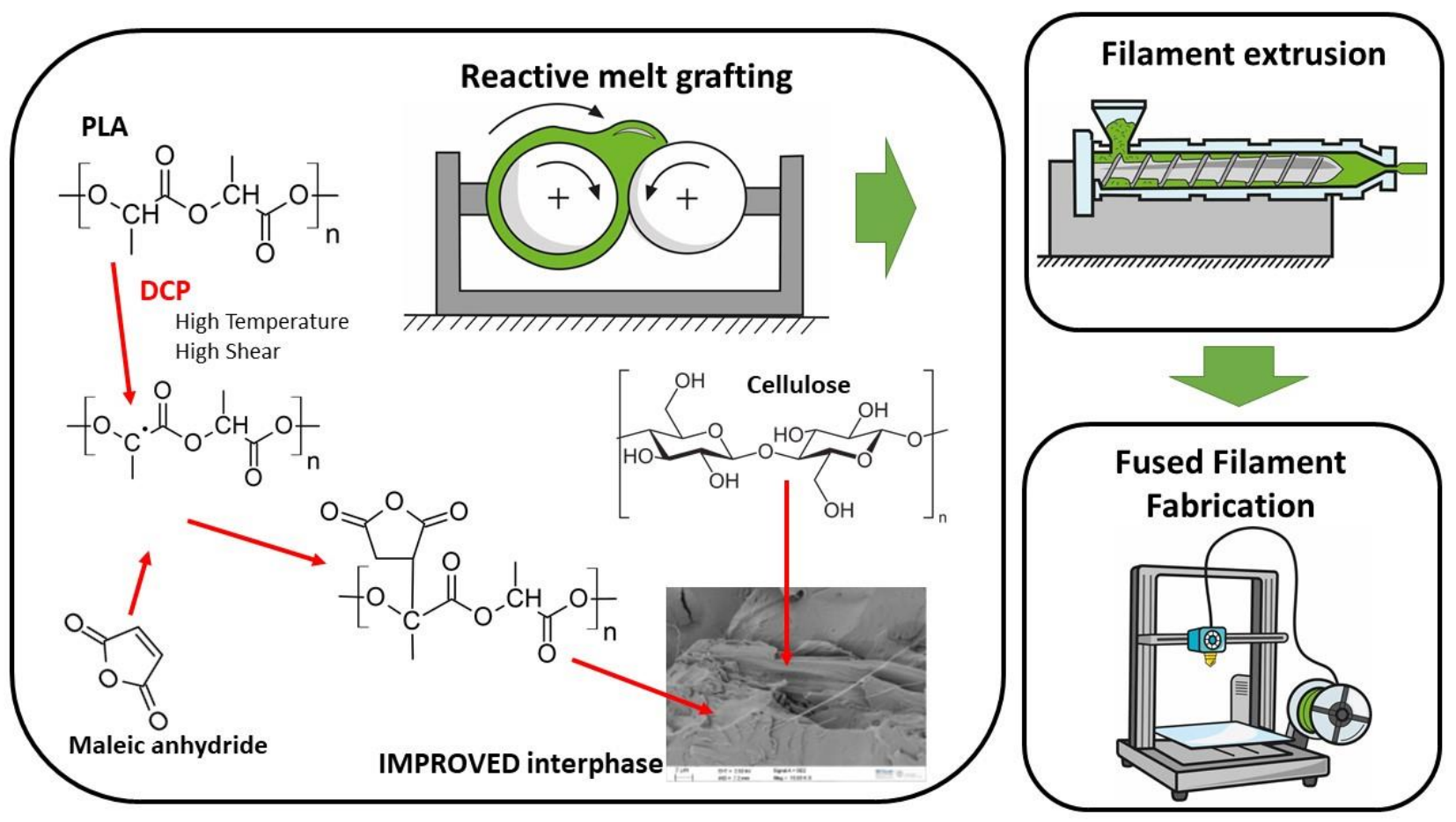

\section{Introduction}

Fused filament fabrication (FFF) is one of the more attractive additive manufacturing (AM) techniques due to the continuous decrease of the related costs, high speed and simplicity of the process [1]. The advantages of FFF over traditional manufacturing processes are the possibility of free design and the absence of a mold in manufacturing thus allowing the realization of complex parts at low costs [2]. FFF is a technique to build up objects layer by layer with the support of computer aided design. A filament is heated inside a nozzle, mounted on a moving system and then extruded in a controlled manner from the bottom layer to the top layer to form the final object [3]. At present, various materials are available for FFF ranging from thermoplastic polymers such as polyamide [4], high-density polyethylene [5], acrylonitrile butadiene styrene (ABS) [6], polylactic acid (PLA), polyetherimide (PEI) [7], thermoplastic polyurethane [8] and polyvinyl alcohol (PVA) [9], to ceramic [10], gypsum, metals, food [11] and even concrete [12]. Polymers based on lactic acid (PLA) are one of the most promising category of plastic materials made from renewable resources due to their good mechanical properties, good workability, excellent barrier properties [1] and biodegradability [13, 14]. PLA is a compostable polymer derived from renewable resources; it has been viewed as a good candidate to reduce the societal solid waste disposal problem. Its low toxicity, along with its environmentally green characteristics, could made PLA an ideal material for food packaging and for other consumer products. Nevertheless, some weak points mainly represented by its low ductility, 
poor toughness, low glass transition temperature, high sensitivity to moisture and relatively low gas barrier should be solved $[15,16]$. The use of natural or synthetic fibers is well contemplated to obtain specific characteristics and major improvements of the properties of polymeric matrices [17]. Natural materials added to a PLA matrix act mostly as a filler to reduce costs without losing the green benefits of a biopolymer [18] or to enhance the biodegradability [19]. The incorporation of natural fillers, characterized by a hydrophilic behavior, into hydrophobic thermoplastic polymers typically results in poor dispersion, high viscosity, and low compatibility. The poor interfacial adhesion between the filler and the matrix generally leads to composites with worse mechanical properties [20]. Compatibilizers are widely used to improve the interface between the fillers dispersed in the polymer matrix. Maleic anhydride (MAH) does not homopolymerize under the reaction conditions used in grafting reactions which makes it a good compatibilizing agent. Free radical melt grafting of maleic anhydride on the polymer chains of polyolefins is an efficient and easy way to improve the compatibility of polymer and different kind of natural fiber from micro- to nanometric scale. Numerous investigations of free-radical grafting of MAH different polyolefins such as: LDPE, HDPE, PP, EPR, EPDM are reported in literature [21]. This reaction is generally achieved when the molten polymer is mixed with MAH and with a peroxide initiator, either in an extruder or in an internal mixer. Grafting was effectively performed in an internal mixer at temperature of $190^{\circ} \mathrm{C}$ and 60 RPM on PLA where dicumyl peroxide was used as initiator at concentration between 0.1 and 0.2 $\mathrm{wt} \%$ and MAH added at concentration between 0.3 and $3 \mathrm{wt} \%[22,23]$. A grafting amount of $0.2-$ $0.5 \%$ was determined by titration analysis, depending on the content of MAH. A concentration of dicumyl peroxide of $1 \mathrm{wt} \%$ resulted to be an optimal formulation to maximize the grafting process and minimize the possibility of undesired side reactions [24, 25]. Moreover, it was underlined that a maleic anhydride content higher than $5 \mathrm{wt} \%$ resulted in no significant improvements [26]. Organic clays such as montmorillonite [23], bentonite and hectorite [27] are used to improve the mechanical properties of composite. Most often, a proper compatibilizer is added to PLA to promote a better dispersion of non-polar organoclay. Maleic anhydride is a common choice to produced high values composites starting from PLA and agricultural wastes. For example, rice husk was an interesting opportunity due to its low cost, renewability, biodegradability and low density. Tsou et al. [28] proposed a possible way to recycle it and incorporate this waste material in PLA to produce green composites through the modification of PLA via reaction grafting in the melting state with MAH. Zhu et al. [29] conducted an extensive investigation on the possibility to use soy protein, an agricultural residue of the soybean oil crushing, as a filler to be compounded with PLA grafted MAH to reduce the total cost while increasing the degradation rate of the composites. The hydrophilic functionalization with maleic anhydride was studied to improve the compatibility between PLA and 
wheat straw by Nyambo et al. [30]. Both PLA and starch are brittle components and lack of chemical compatibility, this results in a brittle composite with low strength, which could be improved by introducing MAH as a compatibilizer as demonstrated by Zhang et al. [31]. They proved that the mechanical properties of PLA/starch composites are improved with just 1wt\% of MAH. In fact, PLA/starch (55/45) composite reached a tensile strength of $52.4 \mathrm{MPa}, 20 \%$ more that the materials without MAH and similar to the neat PLA. Micro cellulose and nanocellulose have been promising evaluated as reinforcing filler for various composites [32]. Specific composition in PLA have been produced either by solution casting up to $20 \%$ of nanocellulose [33], or by melt compounding with $5 \%$ of nanocellulose crystals [34] and with $1 \%$ of microcrystalline cellulose [35]. Moreover, some researchers recently suggested the application of cellulose based composite for 3D printing [9, 36]. PLA has been used to prepare bio-based and biodegradable composites filled with cellulosic materials to reduce the overall costs and improve the mechanical properties [37, 38].

The aim of this work is to study the addition of microcrystalline cellulose into PLA matrix through the grafting of maleic anhydride (MAH) on the PLA backbone as a compatibilizing agent. Filaments composite with increasing amount of MCC are 3D printed with a desktop FFF machine. The mechanical properties, thermal stability and morphology of the resulting composites are characterized and the synergetic effects of the compatibilization and MCC on the mechanical behavior of the composites are evaluated.

\section{Materials and methods}

\subsection{Materials}

Poly(lactic acid) (PLA) grade 4032D with density of $1.24 \mathrm{~g} \mathrm{~cm}^{-3}$ and a melting point of 155 $170^{\circ} \mathrm{C}$ was provided by Nature Works LLC (Minnesota, USA). PLA 4032D is an ideal product for laminations and other packaging applications, due to the excellent optics, good machinability and excellent twist and dead fold. Microcrystalline cellulose, MCC, (specific gravity $=1.56 \mathrm{~g} \cdot \mathrm{cm}^{-3}$, mean molecular weight $=90000 \mathrm{~g} \mathrm{~mol}^{-1}$ ) prepared through the reaction of cellulose with a water solution of strong mineral acid at boiling temperature, supplied by Sigma Aldrich (USA), were selected as microscale reinforcing filler. MCC particles consist of elongated flakes with an average length of about $24 \mu \mathrm{m}$ and a diameter of about $10 \mu \mathrm{m}$ measured with the aid of an optical microscope. Maleic anhydride (MAH) and dicumyl peroxide (DCP) were provided by Sigma Aldrich (USA).

\subsection{Compounding and melt-grafting}

Both PLA pellets and microcrystalline cellulose powder were dried in a ventilated oven at $50^{\circ} \mathrm{C}$ for 24 hours before being processed. PLA with various amounts of microcrystalline cellulose were melt- 
compounded with or without compatibilizer (PLAgMAH) in a Thermo Haake internal mixer at a temperature of $165^{\circ} \mathrm{C}$, with a revolution speed of the rotors of $60 \mathrm{rpm}$ and a processing time of 15 minutes.

Grafting of maleic anhydride (MAH) on the backbone of PLA (PLAgMAH) was performed via radical grafting directly during the melt compounding process with the presence of dicumyl peroxide (DCP) as a radical initiator, following a procedure well described in literature [22]. The PLA was melted for 2 minutes and then DCP and MAH were added at a ratio of 1:10 DCP/MAH and mixed for 3 minutes. The reaction with MAH in quantities from $1 \mathrm{wt} \%$ to $10 \mathrm{wt} \%$ continued during mixing for 5 minutes. Eventually, MCC was added and mixed for another 5 minutes. At the end various, batches of PLA/MMC and PLA/PLAgMAH/MCC were cooled down and grinded at room temperature by IKA grinder machine.

According literature [22], the first step of grafting is the formation of primary radicals via the decomposition of dicumyl peroxide, which then initiates PLA macroradicals by a hydrogen extraction mechanism. The macroradicals subsequently react with maleic anhydride. A possible mechanism of grafting MAH on PLA is schematized in Figure 1.

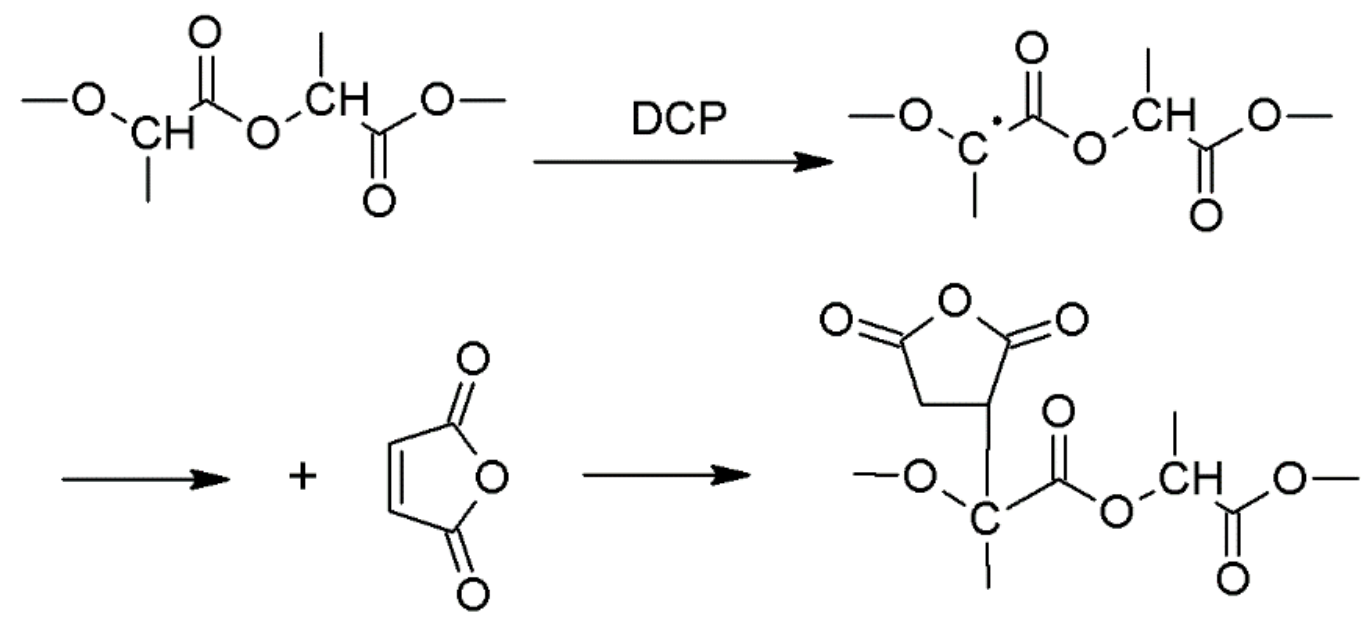

Figure 1. Possible mechanism of grafting MAH on PLA.

\subsection{Filaments extrusion}

PLA compounded with $10 \mathrm{wt} \%$ of MCC, and PLA grafted with $1 \mathrm{wt} \%$ of MAH compounded with $10 \mathrm{wt} \%$ of MCC were grinded and used as masterbatch material to be mixed with neat PLA powder to reach the desired microcellulose concentration in the filaments. The filaments were obtained by using a Next 1.0 (3Devo, Netherlands) single screw extruder. It was characterized by a nozzle of 3 $\mathrm{mm}$ in diameter and an optical encoder to measure the diameter of the extruded filament that allowed an automatic adjustment of the drawing speed to maintain a stable diameter. With this feature, an 
average diameter of $1.75 \pm 0.04 \mathrm{~mm}$ was obtained. A temperature profile of $170^{\circ} \mathrm{C}, 190^{\circ} \mathrm{C}, 185^{\circ} \mathrm{C}$ and $175^{\circ} \mathrm{C}$ in the die zone and a rotation speed of 5RPM were chosen. Neat PLA, PLA compounded with MCC and PLA grafted MAH compounded with MCC filaments containing from $1 \mathrm{wt} \%$ to 10 wt $\%$ of microcellulose were produced via extrusion.

\subsection{Fused filament fabrication}

Sharebot Next Generation desktop 3D printer (Sharebot NG) with a nozzle diameter of 0.35mm was used for the fabrication of dumbbell specimens according to ISO527 1BA standard. A digital 3D model of the samples was built with the aid of the software SolidWorks® and it was exported in STL format. Through the freeware software Slic3r, a G-code file was compiled with the following parameter for the printing: rectilinear type of infill, infill angle $\pm 45^{\circ}$, infill percentage $100 \%$, no raft, layer height $0.2 \mathrm{~mm}$, nozzle temperature $220^{\circ} \mathrm{C}$, bed temperature $40^{\circ} \mathrm{C}$ and deposition rate $40 \mathrm{~mm} / \mathrm{s}$.

Interlayer adhesion of FDM Compact Tension (CT) specimens was investigated through the evaluation of fracture toughness according to ASTM D5045. Their dimensions were $24 \mathrm{~mm}$ $(\mathrm{W}) \times 24 \mathrm{~mm}(\mathrm{H}) \times 5 \mathrm{~mm}(\mathrm{~T})$, with a crack length of $6 \mathrm{~mm}$ as shown in Figure 2a. CT specimens were printed with the same parameters reported before in order to have the crack plane aligned with the layers as visible in Figure 2b. In each specimen, a sharp notch of $9 \mathrm{~mm}$ in length was done by means of a broaching machine equipped with a fresh razor blade (Figure 2c). Specimen were tested in tensile configuration with a self-made interface between specimen and tensile machine.

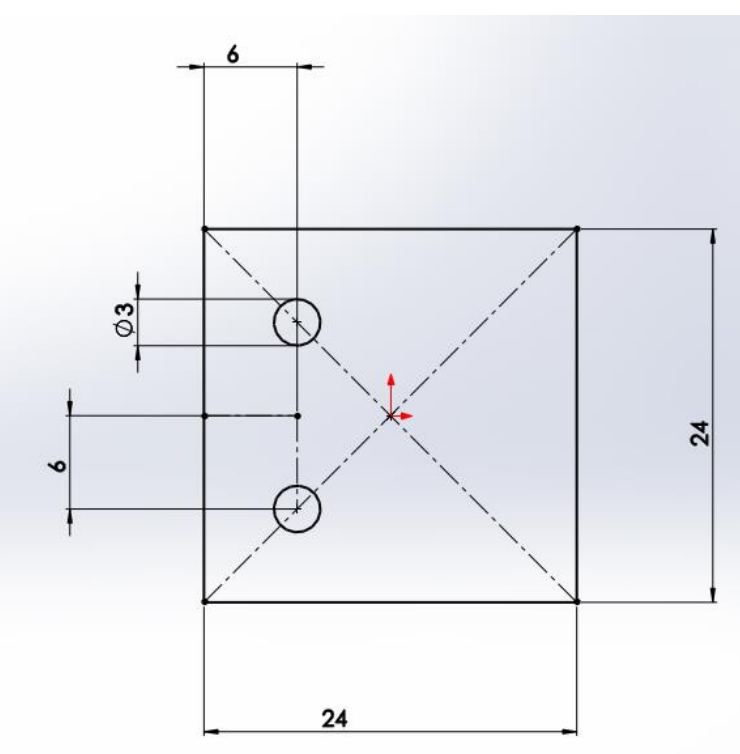

(a)

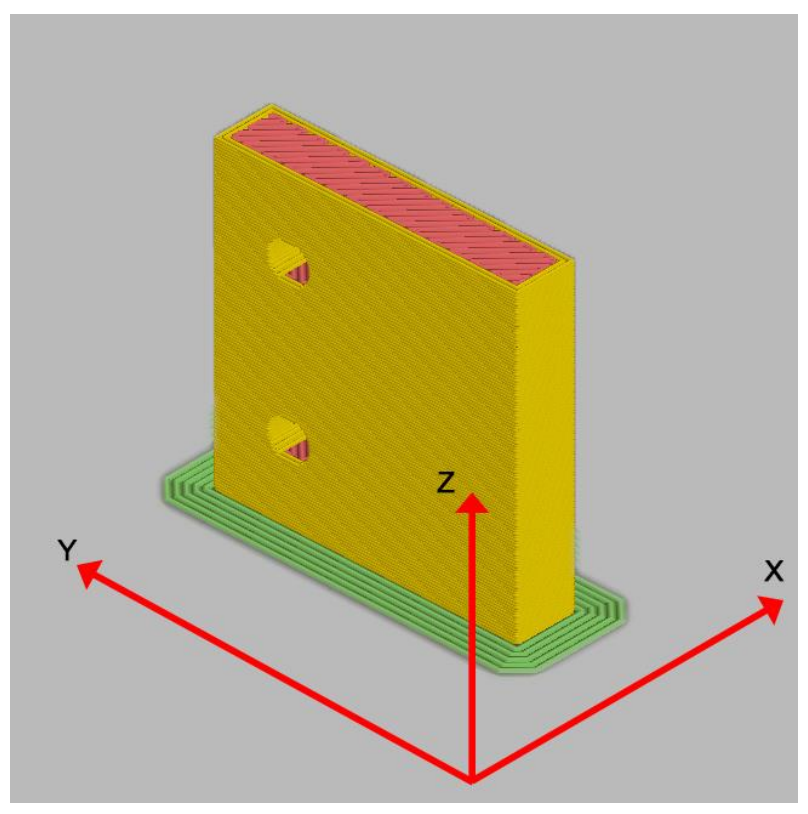

(b) 


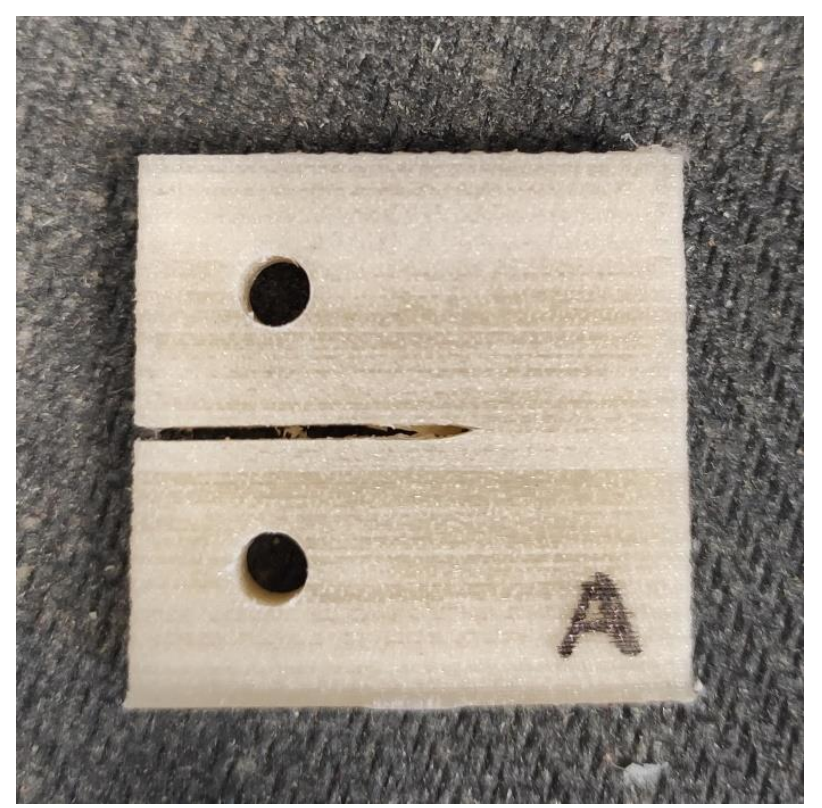

(c)

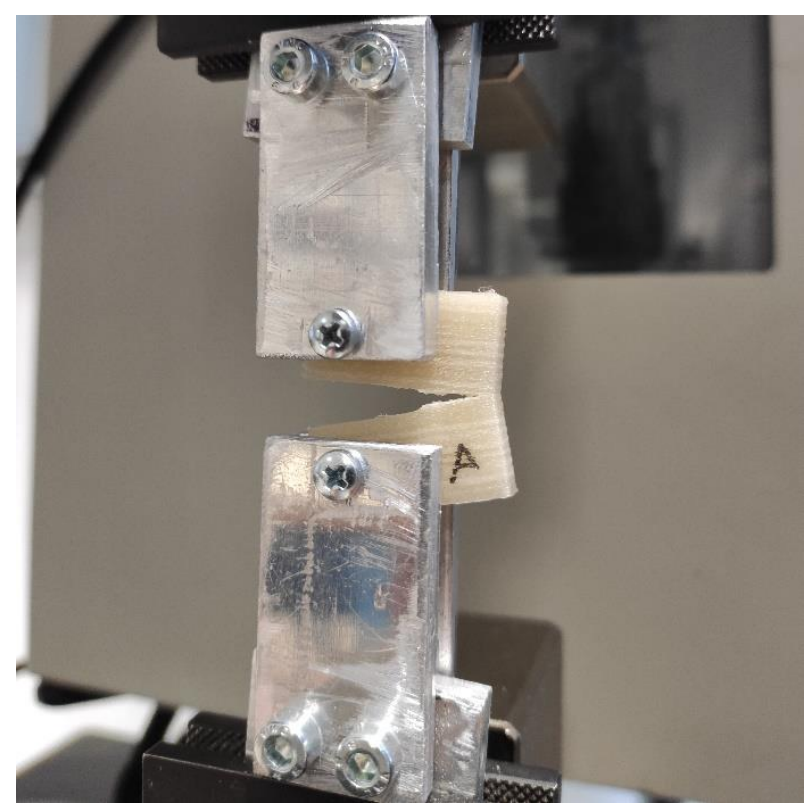

(d)

Figure 2. (a)Graphical picture of the CT specimen as draw in SolidWorks (all the measurements are in $\mathrm{mm}$ ), (b) 3D draw of the specimen during printing, (c) CT specimen after notching and (d) tensile test.

\subsection{Testing techniques}

The grafting reaction of maleic anhydride on PLA was investigated through Fourier-transform infrared spectroscopy (FTIR) analysis carried out by a Spectrum One from Perkin-Elmer in ATR configuration. Spectra were collected in the wavenumber range from 4000 to $650 \mathrm{~cm}^{-1}$ by averaging 4 scans.

Degradative mechanisms of the materials while subjected to a constant heating were studied with thermogravimetric analysis (TGA). The analysis was performed with the help of a TA Instruments TGAQ5000 machine, with a heating rate of $10^{\circ} \mathrm{C} / \mathrm{min}$ in the range between $40^{\circ} \mathrm{C}$ and $700^{\circ} \mathrm{C}$ flushing nitrogen at $15 \mathrm{ml} / \mathrm{min}$.

Viscosity was also measured as a function of shear rate using an Anton Paar MCR 301 rheometer. Before experiments, the samples were dried at $40{ }^{\circ} \mathrm{C}$ for $24 \mathrm{~h}$. The rheometer was operated in the rotational mode with parallel plate geometry at $190^{\circ} \mathrm{C}$, over a shear rate range of 10 to $1000 \mathrm{~s}^{-1}$. Before testing, the samples were kept under vacuum at room temperature for 24 hours to remove completely the humidity. 
Density of the 3D printable filaments was measured with an Archimedes balance in double distilled water at room temperature through a Gibertini E42 analytical balance following ASTM D792 standard.

Microstructural observations of cryo-fractured surfaces of the 3D printed specimens were performed with a Zeiss Supra 40 high resolution field emission scanning electron microscope (FESEM) with an accelerating voltage of $2.5 \mathrm{kV}$. Sample were coated with a thin platinum-palladium conductive layer.

Differential scanning calorimetry (DSC) was performed with a Mettler DSC 30 calorimeter. The tests were performed from $20^{\circ} \mathrm{C}$ to $200^{\circ} \mathrm{C}$ at a heating and cooling rate of $10^{\circ} \mathrm{C} / \mathrm{min}$ under a nitrogen flow of $100 \mathrm{ml} / \mathrm{min}$. Glass transition temperature $T_{g}$, crystallization temperature $T_{c}$, melting temperature $\mathrm{T}_{\mathrm{m}}$ and the degree of crystallinity $\chi_{\mathrm{c}}$ were evaluated. The value of enthalpy for fully crystalline PLA was taken to be $93.6 \mathrm{~J} / \mathrm{g}[39]$.

Dynamic mechanical thermal analysis (DMTA) tests were carried out using a TA Instrument DMA Q800 device, in the temperature range from $-10^{\circ} \mathrm{C}$ to $150^{\circ} \mathrm{C}$, at a heating rate of $3^{\circ} \mathrm{C} / \mathrm{min}$, a strain amplitude of $0.05 \%$ and a frequency of $1 \mathrm{~Hz}$. The storage modulus (E'), the loss modulus (E') and the loss tangent $(\tan \delta)$ were evaluated as a function of the temperature.

Quasi-static tensile tests were performed using an Instron 5969 tensile test machine, equipped with a $50 \mathrm{kN}$ load cell, at room temperature with a crosshead speed of $10 \mathrm{~mm} / \mathrm{min}$. At least five specimens for each composition were tested.

Fracture toughness of the interface between two subsequent layers was determined through a tensile test on CT specimen at $10 \mathrm{~mm} / \mathrm{min}$ according to ASTM D5045 evaluating the stress intensity factor to measure the stress field around the crack tip with equation 1 :

$$
K_{Q}=\frac{P_{Q}}{B W^{1 / 2}} f(x)
$$

$K_{Q}$ is a conditional fracture toughness calculation, $P_{Q}$ is a conditional load identified according to ASTM D5045, $B$ is specimen thickness, $W$ is specimen width, $a$ is the crack length, $x$ is the crack length to specimen width ratio $a / W(0.2<x<0.8)$ and $\mathrm{f}(\mathrm{x})$ is tabulated in ASTM D5045. To validate the linear elastic plane-strain fracture toughness measurement, $\mathrm{P}_{\mathrm{Q}}$ is determined by finding the intersection of the load vs. crack opening curve and a line having a compliance $5 \%$ greater than the initial slope of the curve. $\mathrm{P}_{\max }$ is then taken to be the maximum load sustained by the specimen. Under the following conditions, $\mathrm{K}_{\mathrm{Q}}$ is equivalent to $\mathrm{K}_{\mathrm{IC}}$ : 


$$
\begin{gathered}
\frac{P_{M A X}}{P_{Q}}<1.1 \\
B, a,(W-a)>2.5\left(\frac{K_{Q}}{\sigma_{y}}\right)^{2}
\end{gathered}
$$

where $\sigma y$ is the yield strength of PLA, which is taken to be $55 \mathrm{MPa}$ based upon previous material testing.

The results of mechanical tests were evaluated using ANOVA with the software R-Cran, considering the quantity of MCC, MAH and their interaction. The $P$ value was used as a tool to check the significance of the examined variables. A $P$ value lower than 0.05 (i.e. 95\% confidence interval) indicates that the examined parameter is significant.

The samples coding based on the composition is summarized in Table 1. For example, a sample coded PLAgMAH_05MCC indicates the presence of 5wt\% of MCC in a matrix composed of PLA grafted with MAH. According to the preparative methods discussed above, the content of MAH is in all cases fixed at $1 \mathrm{wt} \%$ of MCC.

Table 1. Sample code, melt flow of materials, and final composition of filaments and 3D printed parts.

\begin{tabular}{lcccc}
\hline Code & $\begin{array}{c}\text { PLA } \\
(\mathrm{wt} \%)\end{array}$ & $\begin{array}{c}\text { MCC } \\
(\mathrm{wt} \%)\end{array}$ & $\begin{array}{c}\text { MAH } \\
(\mathrm{MCC} w \mathrm{wt} \%)\end{array}$ & $\begin{array}{c}\text { DCP } \\
(\mathrm{MAH} w \mathrm{wt})\end{array}$ \\
\hline PLA & 100 & 0 & 0 & 0 \\
PLA_01MCC & 99 & 1 & 0 & 0 \\
PLAgMAH_01MCC & 98.89 & 1 & 0.1 & 0.01 \\
PLA_03MCC & 97 & 3 & 0 & 0 \\
PLAgMAH_03MCC & 96.67 & 3 & 0.1 & 0.01 \\
PLA_05MCC & 95 & 5 & 0 & 0 \\
PLAgMAH_05MCC & 94.45 & 5 & 0.1 & 0.01 \\
PLA_10MCC & 90 & 10 & 0 & 0 \\
PLAgMAH_10MCC & 88.9 & 10 & 0.1 & 0.01 \\
\hline
\end{tabular}

\section{Results and discussions}

FTIR is an effective technique to analyze the structures of maleated polymers. In fact, the presence of MAH grafted on PLA backbone can be identified by comparing the FTIR spectra of PLA filaments extruded with and without MAH and DCP (see Figure 3). Comparing the spectra of neat PLA and the ones of PLA grafted with maleic anhydride, it is possible to observe the presence of new absorption band, in particular at: $695 \mathrm{~cm}^{-1}$ corresponding to cis-vinyl C-H bending [22]; $820 \mathrm{~cm}^{-1}$ corresponding 
to the out of plane deformation for carboxyl groups from MAH [40]; $1635 \mathrm{~cm}^{-1}$ corresponding to the cyclic C-C stretching that might be a confirmation of the chemical interaction between PLA and MAH [26, 40]; $1790 \mathrm{~cm}^{-1}$ (appearing as a shoulder due to the overlapping with the very large peak of the carbonyl $>\mathrm{C}=\mathrm{O}$ stretching of PLA at around $1748 \mathrm{~cm}^{-1}$ ) and $1850 \mathrm{~cm}^{-1}$ are assigned to the symmetric and asymmetric stretching of the carbonyl groups of the saturated cyclic anhydride ring of MAH respectively [22, 24, 26, 41]. The absorption peaks are visible only for the highest amount of MAH which is due to the low MAH in the other two samples (1wt\% and 5wt\%) [41]. 


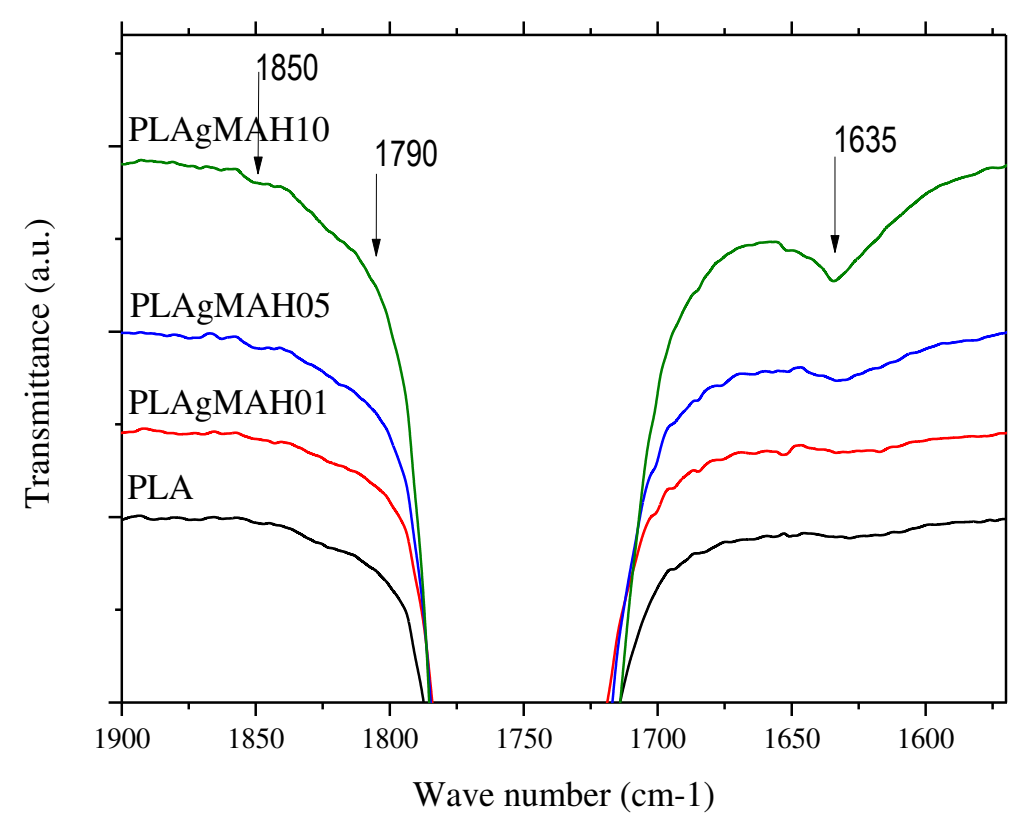

(a)

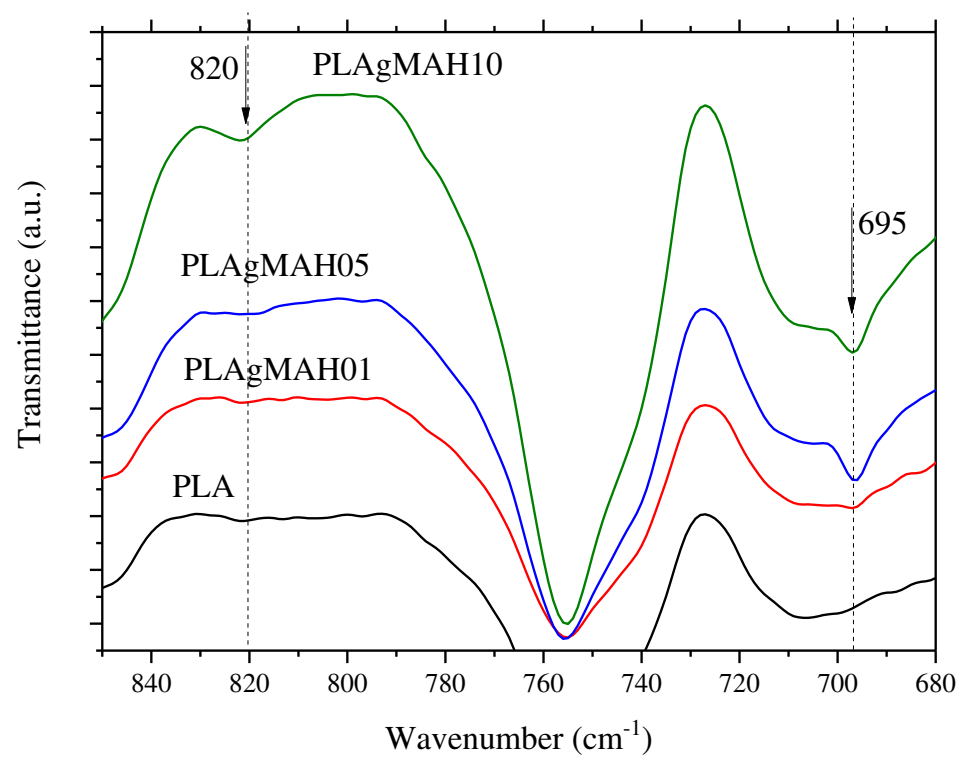

(b)

Figure 3. FTIR spectra for neat PLA and selected PLA grafted MAH focusing on the peaks related with the grafting of MAH.

Thermal degradation behavior of the micro composite materials was investigated through thermogravimetric analysis on filaments specimens and the thermograms are reported in Figure 4. 


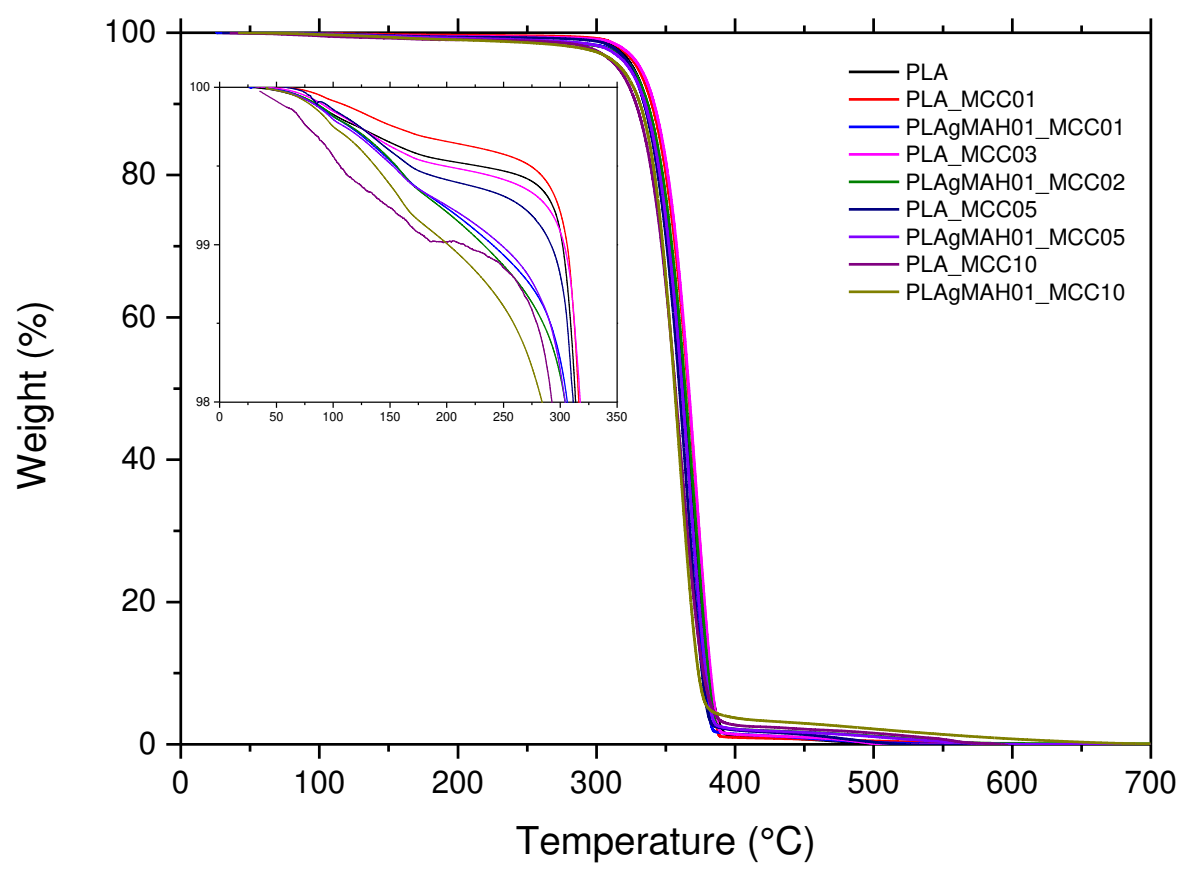

(a)

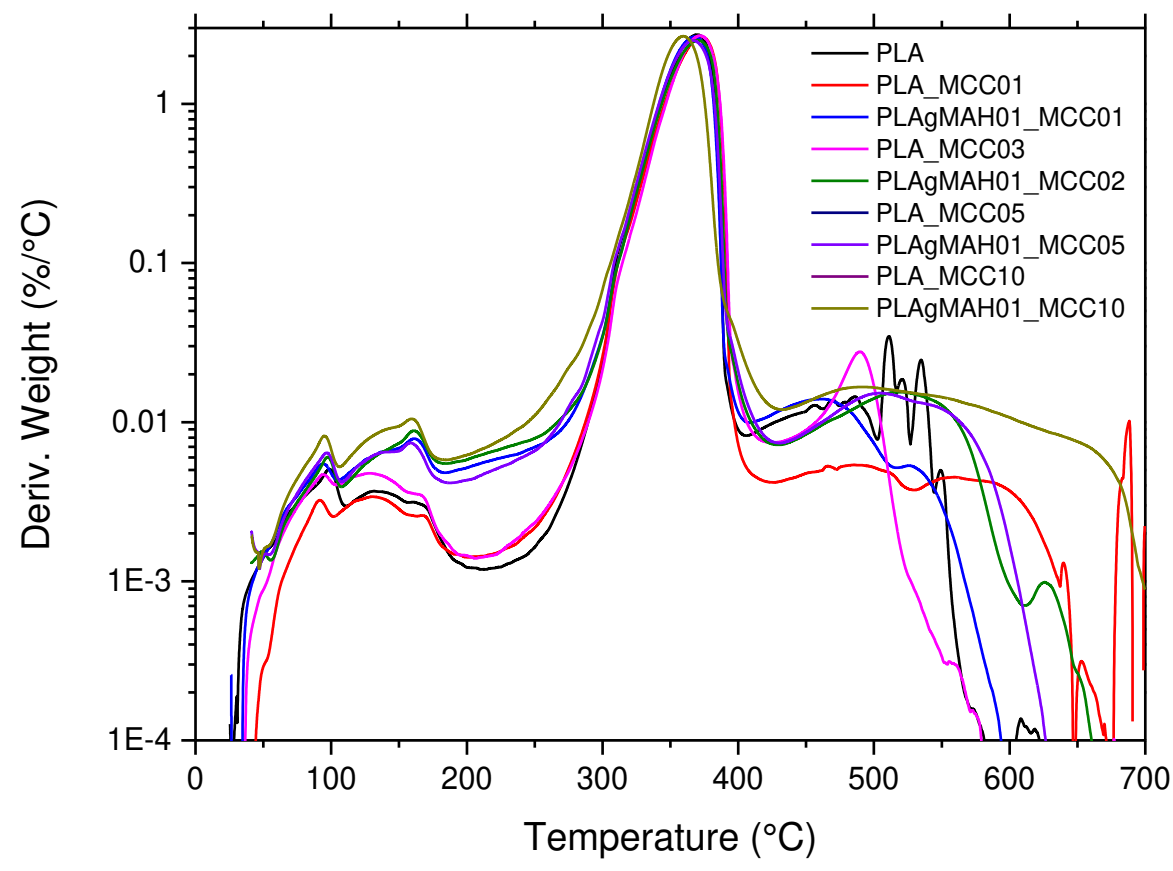

(b)

Figure 4. Thermogravimetric curves (a) and derivative curves (b) of neat PLA compared with PLA grafted MAH with different amount of MAH and MCC.

In particular, as reported in Figure $4 a$, neat PLA is characterized by a single degradation step centered at $370^{\circ} \mathrm{C}$. The onset decomposition temperature of neat PLA is found to be around $315^{\circ} \mathrm{C}$. The decomposition temperature of the samples was affected by the addition of MAH. Moreover, the weight loss started at around $290^{\circ} \mathrm{C}$ for MAH grafted samples, and this could be correlated with the 
lower molecular weight of PLA when it is MAH treated [22]. An initial weight loss, between 0.5\% and $1 \%$, was found for all the filaments and, according to the derivative curve peak at $100^{\circ} \mathrm{C}$, this could be related to the evaporation of residual water. For samples with the highest amount of MCC, a small peak in the derivative thermogravimetric curves was detected at around $160^{\circ} \mathrm{C}$. This peak could correspond to the degradation of the small oligomeric chains formed during the melt compounding process.

Viscosity is a key parameter in extrusion-based AM systems in which the polymer must be first transformed in a filament which is then melted inside a nozzle prior to be deposited on the building plate to form the desired object. The addition of MCC slightly decreased the viscosity of biocomposites up to $3 \%$ of microcrystal cellulose with or without PLAgMAH. It is also worth noting the synergistic effect of MAH on viscosity of composites at 5\% and especially at $10 \%$ of MCC. Furthermore, a broader view of the combined effect of MCC and MAH on the apparent viscosity as a function of the shear rate can be correspondingly appreciated in Figure 5.

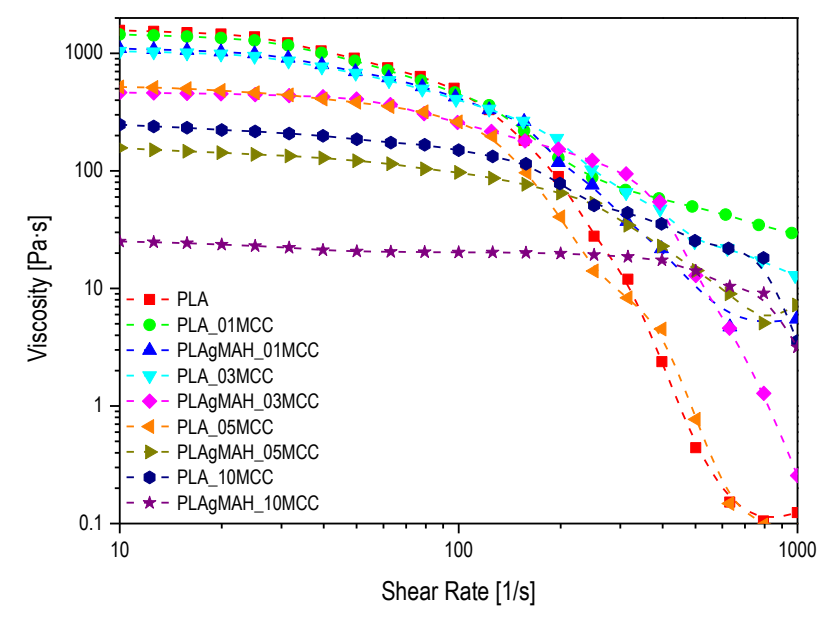

Figure 5. Viscosity values for filaments made of PLA and PLA grafted MAH in function of the content of MCC.

Biocomposites at $1 \%$ and 3\% of MCC behaved similarly to PLA at low shear rate but exhibited higher viscosity at higher shear rate. On the other hand, a significant and progressive reduction of viscosity could be observed for compositions above 3wt\% of MCC, in PLA and PLAgMAH, and the decrease appeared more rapid for samples with MAH at low shear rate. This behavior could be attributed to the presence of micrometric fillers with a low area over volume ratio and due to the poor interaction between MCC and neat PLA for samples without MAH. Despite the improved interaction between PLA and MCC given by MAH, viscosity of biocomposites at 5\% and $10 \%$ of MCC decreased furtherly, due to a possible decrease in molecular weight of PLA after the reaction with DCP that 
hinders the improved matrix - filler compatibility, in particular for, as reported in similar studies [22, 24]. The shift of the viscosity curves towards lower values and the fading of the shear-thinning behavior could be correlated with a decrease in the molecular weight as reported by Cooper-White et al. [42] for PLLA with decreasing molecular weights. A similar behavior was observed by Barczewski et al., in fact they found an increase in MFI of PLA biocomposites with the increasing content of lignocellulosic filler, i.e. micrometric grinded nutshell [43].

Figure 4 shows a shear thinning behavior for neat PLA and microcomposite materials without MAH above $100 \mathrm{~s}^{-1}$. Shear rates in the nozzle of a FFF machine are commonly in the range of $100-200 \mathrm{~s}^{-1}$ [44], so the decrease in viscosity played by the incorporation of MCC and the shear thinning behavior around $100 \mathrm{~s}^{-1}$ appeared beneficial effects for the printability of these biocomposite filaments. On the other hand, the compounding of MCC with PLAgMAH determined a significant reduction of viscosity at low shear rate, but in the processing zone at about $200 \mathrm{~s}^{-1}$ the slight increase of viscosity was not considered critical for 3D printing. In the case of PLAgMAH_10MCC the effect of viscosity decreased due to DCP seemed to be compensated by the addition of MCC in almost all the range of shear rate.

Thermal properties of the PLA, PLA/MCC and PLA grafted MAH/MCC composite filaments were determined using differential scanning calorimetry (DSC) and the thermograms are reported in Figure 6. The glass transition temperature, the crystallization and melting temperatures, the crystallization and melting enthalpies and degree of crystallinity obtained from the DSC are reported in Table 2. Glass transition is a complex property which is related to several factors including intermolecular interactions, steric effects, chain flexibility, molecular weight, branching and cross-linking density. Due to the poor compatibility between cellulose and PLA, glass transition is not expected to change upon the introduction of MCC [45, 46], but Tg could decrease by the presence of maleic anhydride grafted on PLA, due to an increased mobility of the polymeric chains [41]. The improved chain mobility promoted by MAH and the nucleating effect of MCC lowered the crystallization temperature [47]. Crystallization temperature (Tc) and crystallinity fraction (Xc) are affected by the introduction of microcellulose and maleic anhydride. Maleic anhydride increases the crystallinity fraction with respect to the corresponding PLA-MCC composite filament. The melting temperature of neat PLA crystallites is identified at around $168^{\circ} \mathrm{C}$ and slightly increased with the presence of filler. From this increase in melting temperature, MCC appeared to affect the crystalline structure of PLA in terms of size and perfection of the crystalline lamellae of PLA [48]. The maximum amount of crystallization, according to the crystallization enthalpy $(\Delta \mathrm{Hc})$, is observed for the sample loaded with 1,3 and 5 wt $\%$ of MCC in the presence of MAH. This confirms the previous observations related to the role of 
cellulose as nucleating agents for PLA $[46,49]$. The maximum of crystallizability $X_{\max }$ could be calculated by the enthalpy of melting peak, as reported in Table, and it showed an increase from about $26 \%$ of pristine PLA, to about $39-45 \%$ for MCC biocomposite. The higher the content of MCC, the higher the crystallizability, and even higher in the presence of PLAgMAH.

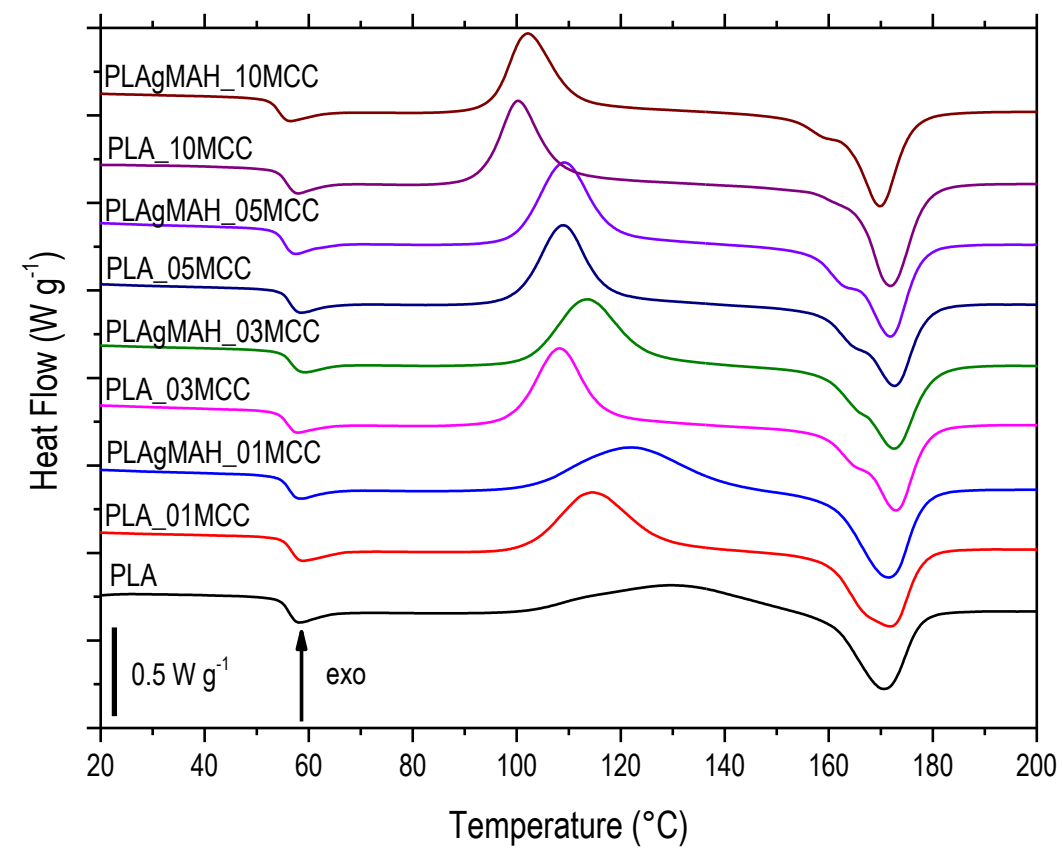

(a)

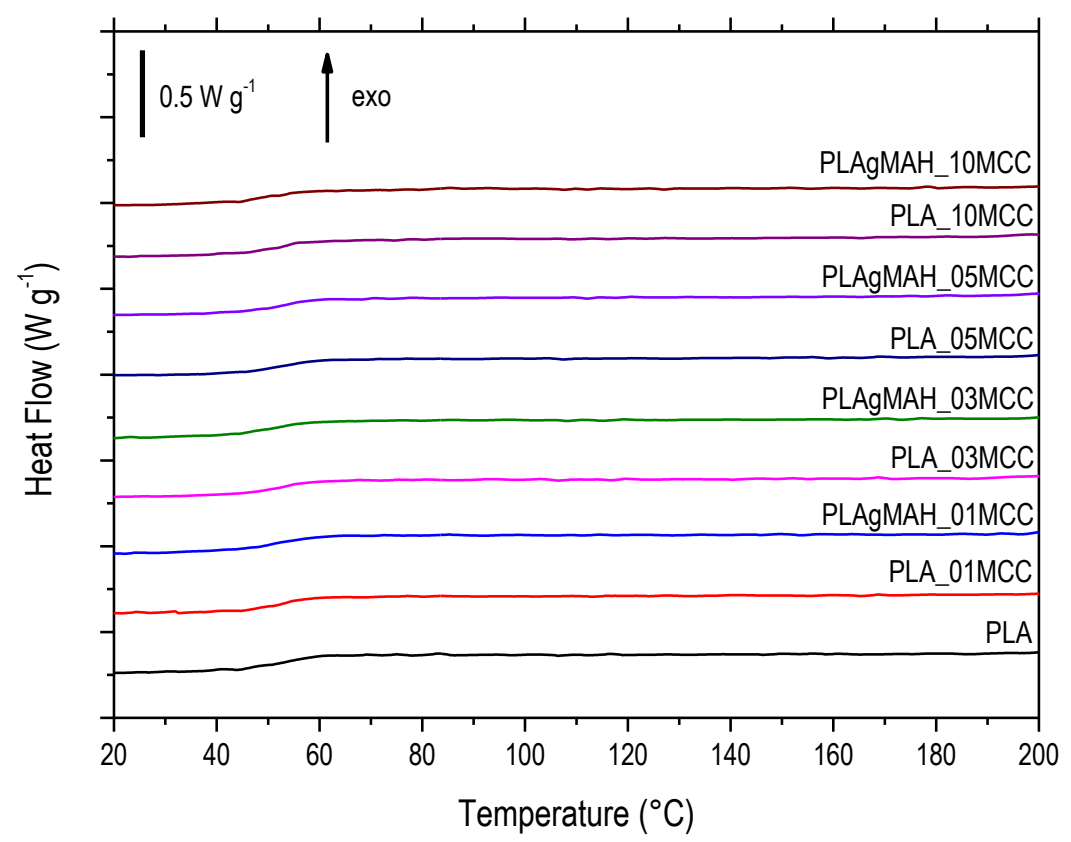

(b)

Figure 6. Representative DSC curves for filaments made of PLA / MCC and PLA grafted MAH and MCC, first heating (a) and cooling (b) scan. 
Table 2. Crystallinity degree according to first scan and glass transition temperature, crystallization temperature, melting temperature for PLA and relative microcomposite filaments from DSC curves. *max crystallizability calculated from the melting peak.

\begin{tabular}{lccccccc}
\hline & $\begin{array}{c}\mathrm{Xc} \\
\%\end{array}$ & $\begin{array}{c}\mathrm{Tg} \\
{ }^{\circ} \mathrm{C}\end{array}$ & $\begin{array}{c}\mathrm{Tc} \\
{ }^{\circ} \mathrm{C}\end{array}$ & $\begin{array}{c}\Delta \mathrm{Hc} \\
\mathrm{J} \mathrm{g}^{-1}\end{array}$ & $\begin{array}{c}\mathrm{Tm} \\
{ }^{\circ} \mathrm{C}\end{array}$ & $\begin{array}{c}\Delta \mathrm{Hm} \\
\mathrm{J} \mathrm{g}^{-1}\end{array}$ & $\begin{array}{c}\mathrm{X}_{\max }{ }^{*} \\
\%\end{array}$ \\
\hline PLA & 1.1 & 59.2 & 134.0 & 23.0 & 168.0 & 24.0 & 25.6 \\
PLA_01MCC & 1.7 & 59.4 & 120.5 & 34.2 & 167.2 & 35.8 & 38.6 \\
PLAgMAH_01MCC & 0.6 & 59.5 & 128.1 & 36.8 & 168.5 & 37.4 & 40.4 \\
PLA_03MCC & 0.9 & 59.5 & 113.4 & 35.4 & 170.4 & 36.2 & 39.9 \\
PLAgMAH_03MCC & 0.2 & 59.2 & 117.2 & 37.1 & 170.4 & 37.3 & 41.2 \\
PLA_05MCC & 1.1 & 59.1 & 113.6 & 34.6 & 170.3 & 35.6 & 40.0 \\
PLAgMAH_05MCC & 2.0 & 58.6 & 113.2 & 37.0 & 169.5 & 38.8 & 43.9 \\
PLA_10MCC & 3.0 & 59.0 & 108.2 & 32.3 & 169.7 & 34.8 & 41.3 \\
PLAgMAH_10MCC & 3.1 & 57.3 & 108.2 & 34.4 & 167.8 & 37.0 & 44.5 \\
\hline
\end{tabular}

Storage modulus curves of 3D printed specimen as a function of temperature are shown in Figure 7a. In the glassy zone below the glass transition temperature, PLA exhibits value of E' (about $1300 \mathrm{MPa}$ at $23^{\circ} \mathrm{C}$ ) lower than that of biocomposite. In particular, the addition of micro cellulose pushes $\mathrm{E}^{\prime}$ towards $1450 \mathrm{MPa}$ at $23^{\circ} \mathrm{C}$ in the case of PLA_10MCC. The grafting of maleic anhydride boosts the stiffening effect of MCC for all the composition, and the value of E' at $23^{\circ} \mathrm{C}$ of PLAgMAH_10MCC reaches $1480 \mathrm{MPa}$. At temperatures that correspond to the glass transition temperature as revealed by DSC, it is possible to observe a drop in the E' value that seems to match the decrease in $\mathrm{Tg}$ found in DSC for as concerns the shift of this drop towards lower temperature. Above $100^{\circ} \mathrm{C}$ storage modulus starts to increase again due to crystallization process and also in this case the curves are shifted to left with an increased amount of micro-cellulose due to a possible nucleating effect or a more favorable chain mobility promoted by the viscosity decrease. Figure $7 b$ shows loss factor $(\tan \delta)$ curves as a function of temperature for 3D printed specimen. Neat PLA exhibits the lowest damping peak $\left(2.07\right.$ at $\left.74^{\circ} \mathrm{C}\right)$ between all the tested composition, but no trend can be evidenced with the increase of MCC or with the presence of MAH. However, all the peaks related to micro-composite material are higher than the one of neat PLA, resulting in the range 2.2-2.6. This effect highlights a slightly better damping performance when micro-cellulose is added to the PLA matrix. 


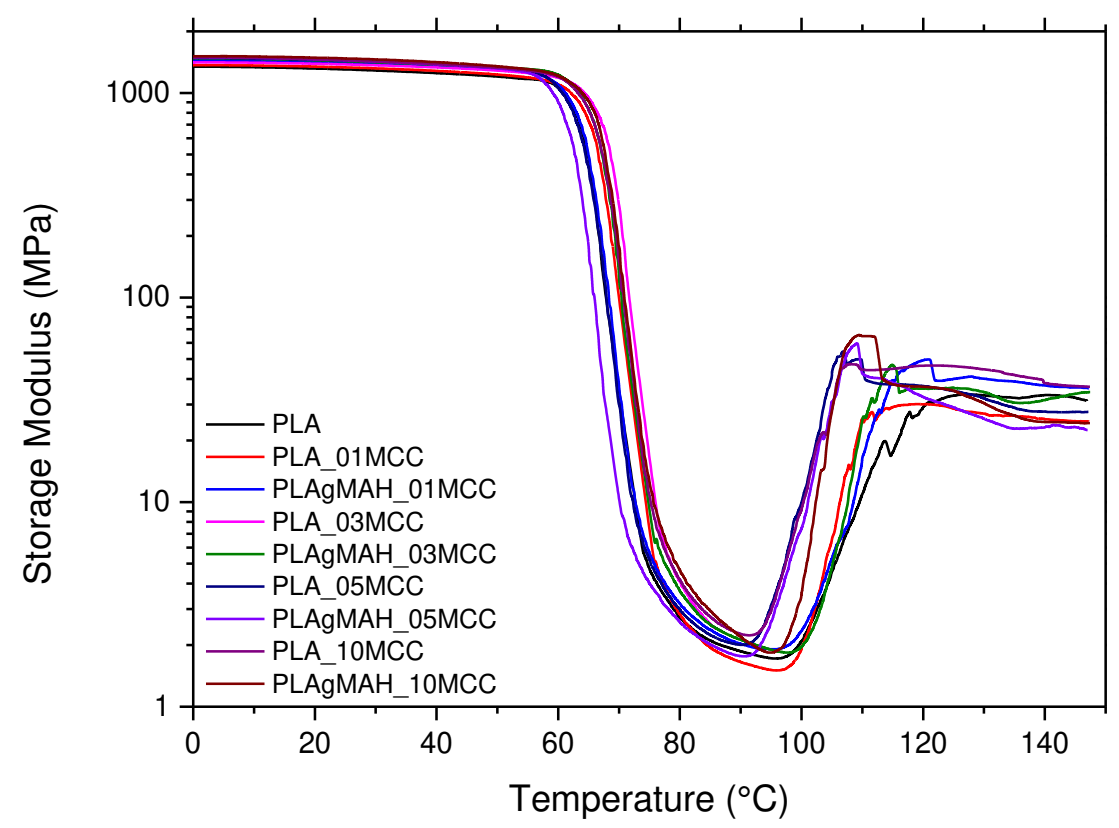

(a)

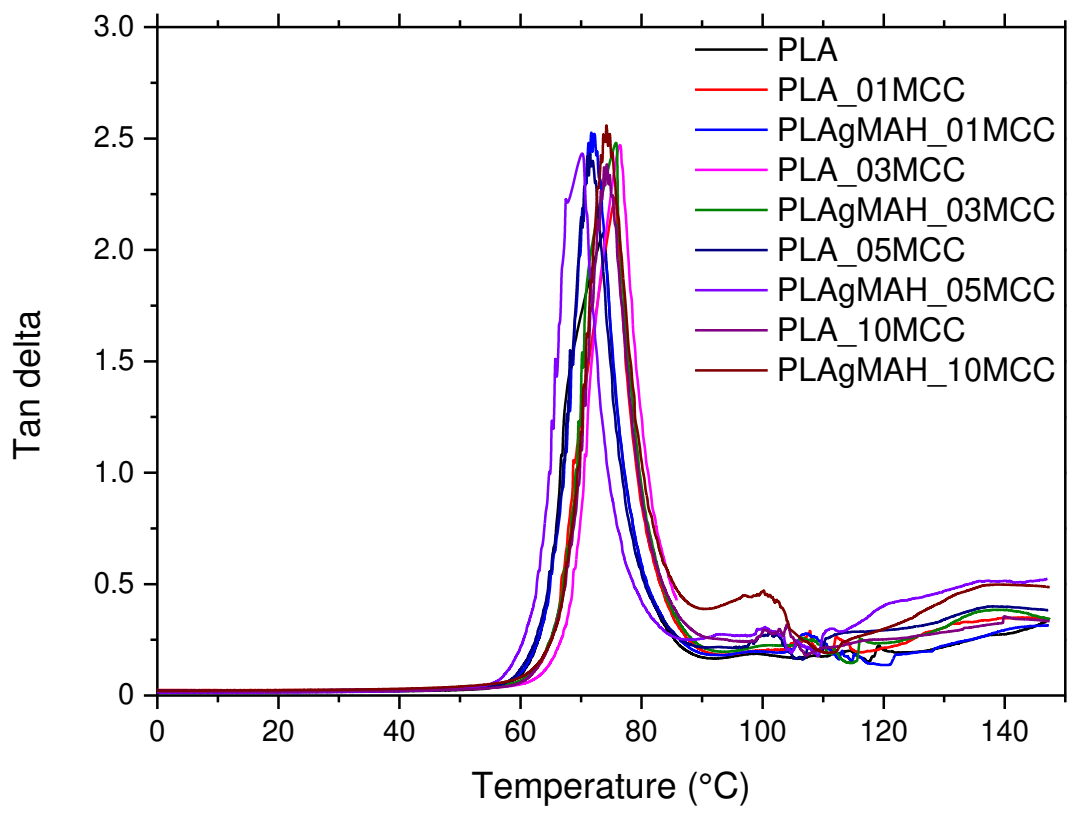

(b)

Figure 7. Storage modulus (a) and tan delta (b) curves for 3D printed specimen of PLA and relative micro composite.

Mechanical properties from quasi-static tensile test on filaments and 3D printed specimen are summarized in Figure 8 and Figure 9, respectively. MCC was found to significantly (p-value $=2.1$ $10^{-6}$ ) increase the elastic modulus of 3D printable filaments. Maleic anhydride had a lower effect on 
elastic modulus ( $\mathrm{p}$-value $=0.014$ ), it is possible to appreciate a higher stiffening effect with an increasing amount of MCC for the samples loaded with MAH. This effect could be explained by an improved interface between the MMC filler and the PLA matrix, but also by a slight increase in the crystallinity of the material as previously seen in DSC and due to a plasticizing effect of MAH. However, this stiffening effect was not translated to 3D printed samples where no effect of MCC was appreciable. The variation in the elastic modulus of 3D printed samples, as highlighted by ANOVA, is slightly affected by the interaction between MCC and MAH (p-value $=0.062$ ). The statistically significative difference between 3D printed samples made of PLA grafted or not with MAH could be due to the increase of the crystallinity fraction or by the enhanced printability and so the density of the specimen.

In fact, it is important to state that the increasing the amount of micro cellulose inside the PLA could impair the printability of these composite due to the clogging of the nozzle during printing and so the easiness to print [36]. Stress at break for filaments was negatively influenced by the presence of both MCC and MAH. MCC inside the polymer matrix acts as a geometrical defect, where the stress could become significantly greater than the surrounding region and leading to a premature failure of the component. A low p-value $\left(<2.210^{-16}\right)$ for as concern the interaction between MAH and MCC indicates that MAH act as a weak and brittle interphase between PLA and MCC. This was particularly evident for the sample with the higher amount of MCC and MAH (PLAgMAH_10MCC). MCC and MAH are found to negatively affect the strain at break of the filaments. The embrittlement of composite material based on PLA with the introduction of MCC is in accordance with other studies in literature $[19,50]$. The different structure of FFF specimen respects to a single filament led to different fracture mechanisms. It is possible to state that the energy at break could not consumed just by the fracture of the single strands that compose the objects but also by the debonding processes intra- and inter-layers [9]. A noticeable effect was found for the filler ( $p$-value $=0.019$ ) however the main reinforcing effect was given by the interaction of MCC and MAH (p-value $=7.410^{-4}$ ). Stress at break increased from 31.2 $\pm 5.1 \mathrm{MPa}$ of the sample printed with neat PLA to 40.7 $\pm 0.8 \mathrm{MPa}$ of PLA grafted MAH with 5wt\% of MCC. According to rheological observations, the decrease in viscosity could have enhanced the adhesion of subsequent layers and adjacent strands. As seen for filament samples, it is possible to detect a decrease in strain at break with the increasing amount of MCC (pvalue $=3.110^{-11}$ ) and this is particular evident when MAH was added due to its brittle behavior (pvalue $=0.03$ ) 

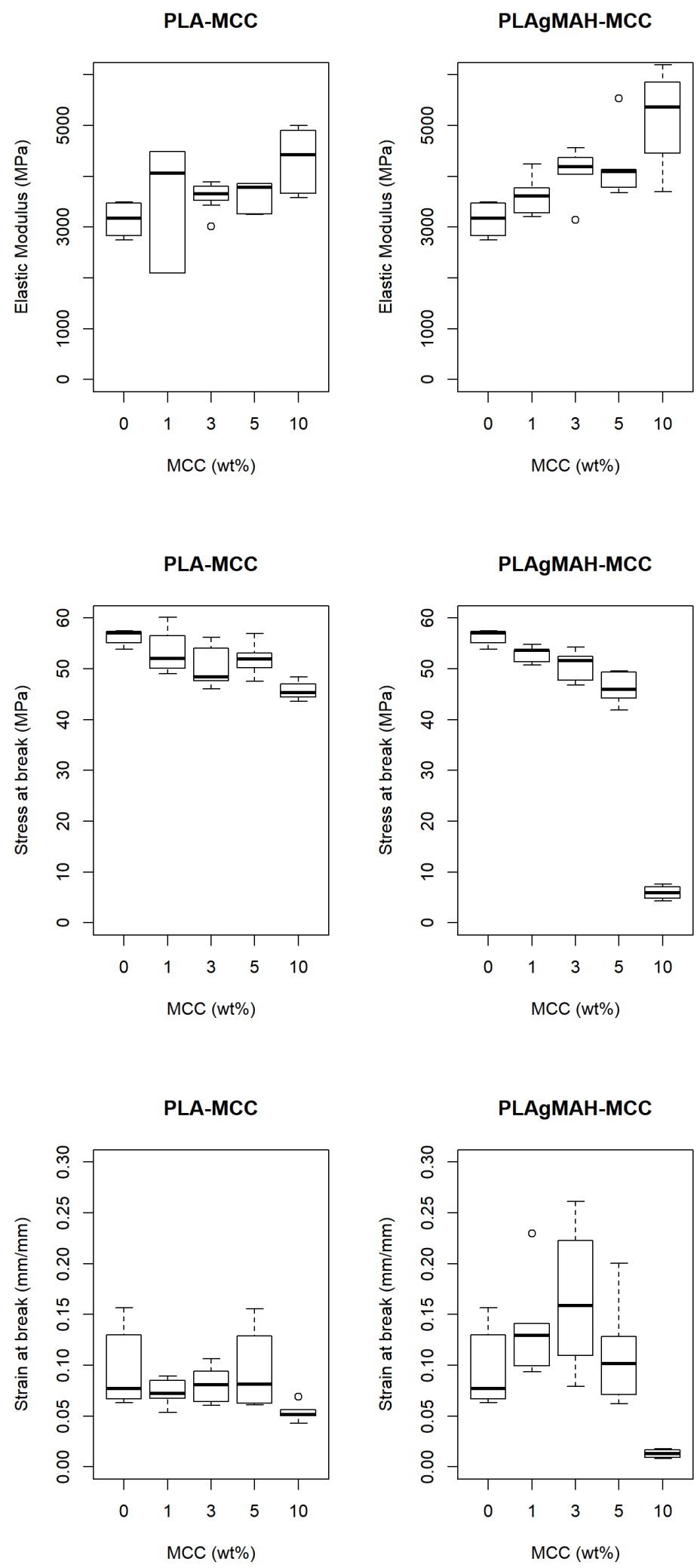

Figure 8. Boxplot of the main mechanical properties related to microcomposite filaments as a function of wt\% of MCC for PLA and PLA grafted MAH. 

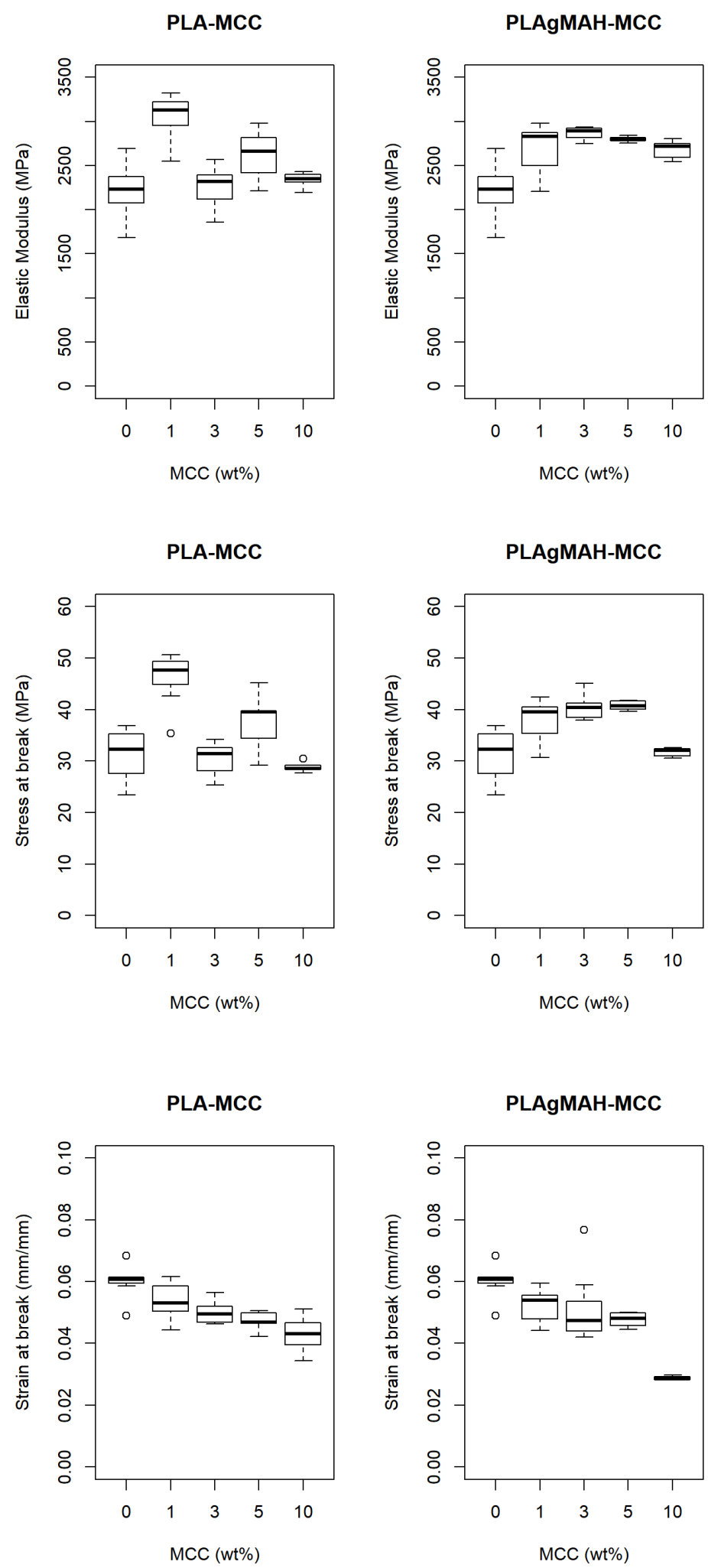

Figure 9. Boxplot of the main mechanical properties related to $3 D$ printed specimen as a function of wt\% of MCC for PLA and PLA grafted MAH. 
The cryogenic fracture surfaces of compression molded specimen produced with PLA and PLA grafted MAH with 5wt\% of MCC are shown in Figure 10. In Figure 10a a very weak interface between the neat polymer matrix and the micro-cellulose can be observed. This behavior could be attributed to a poor chemical interaction between the hydrophobic PLA and the hydrophilic cellulose. Maleic anhydride resulted to be very effective in improving the filler - matrix adhesion. In fact, as it can be appreciated in Figure 10b, a positive enhancement is clearly evidenced with just a low amount (1wt\%) of MAH grafted to the PLA.

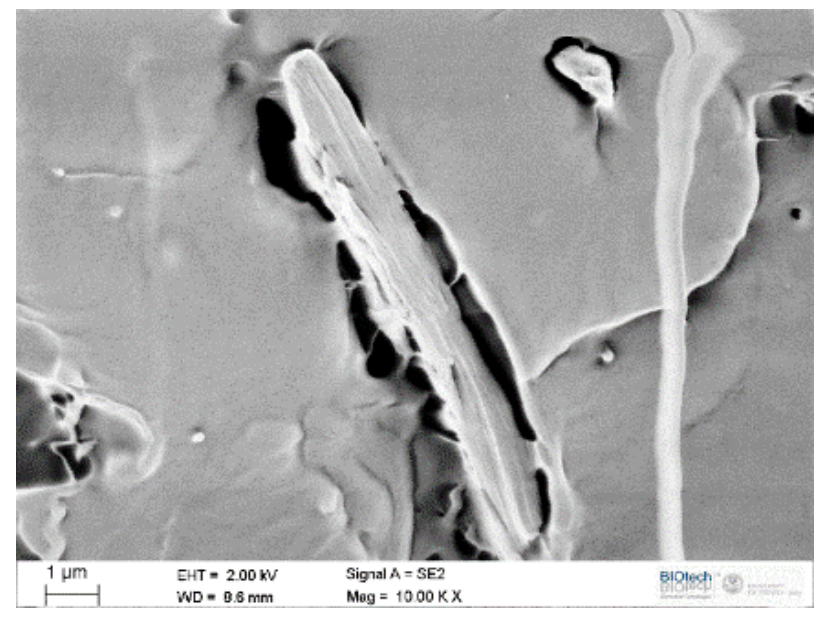

(a)

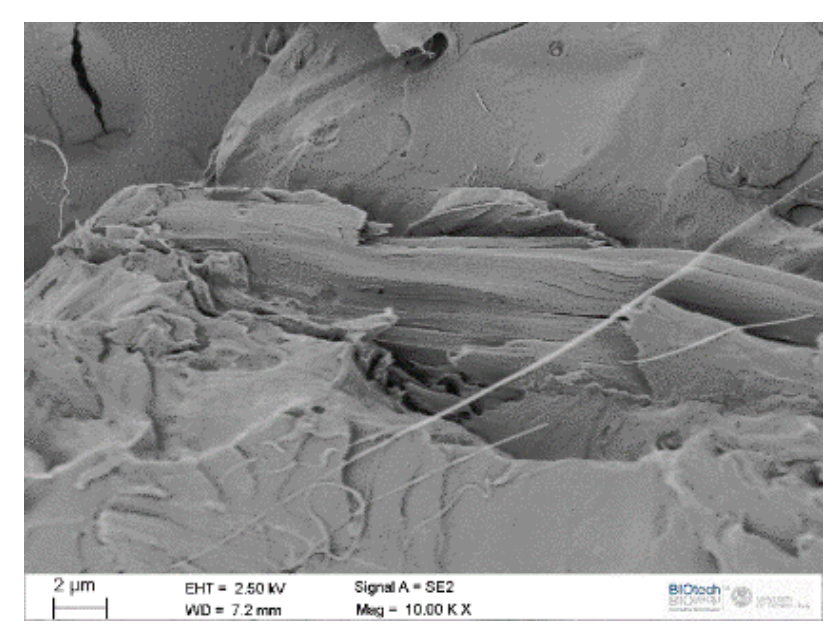

(b)

Figure 10. SEM pictures of cryo fractured surface of PLA_O5MCC (a) and PLAgMAH_MCCO5 (b) compression molded sheets evidencing a good improvement of the interphase between microcellulose and PLA upon the melt grafting of maleic anhydride.

The fracture surface of 3D printed specimen after tensile test with increasing amount of microcellulose with and without the presence of maleic anhydride grafted on the PLA backbone are shown in Figure 11. It is possible to notice the different mechanical response to tensile stress with the increasing amount of filler dispersed in the matrix. Neat PLA and PLA with the lower amount of MCC (Figure 11 a-b) showed an extensive energy absorption with the decohesion of the filaments.

At the highest concentration of filler, 5 and 10wt.\% (Figure $11 \mathrm{c}-\mathrm{f}$ ), the fracture surface become flatter without the evidence of a macroscopic breakage possibly due to the decrease in viscosity that favors viscous flow thus enhancing the inter- and intra-layer adhesion. Filaments broke transversally to the applied stress without detaching from each other. Also, for 3D printed samples without the presence 
of MAH, it is possible to remark a poor compatibility between cellulose and PLA that led to a presence of a large amount of porosity inside the 3D printed filaments.

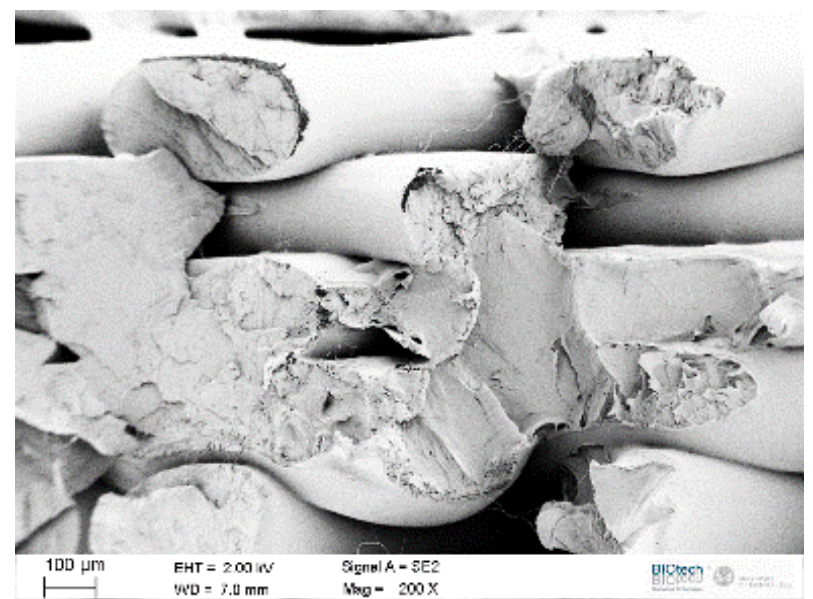

(a)

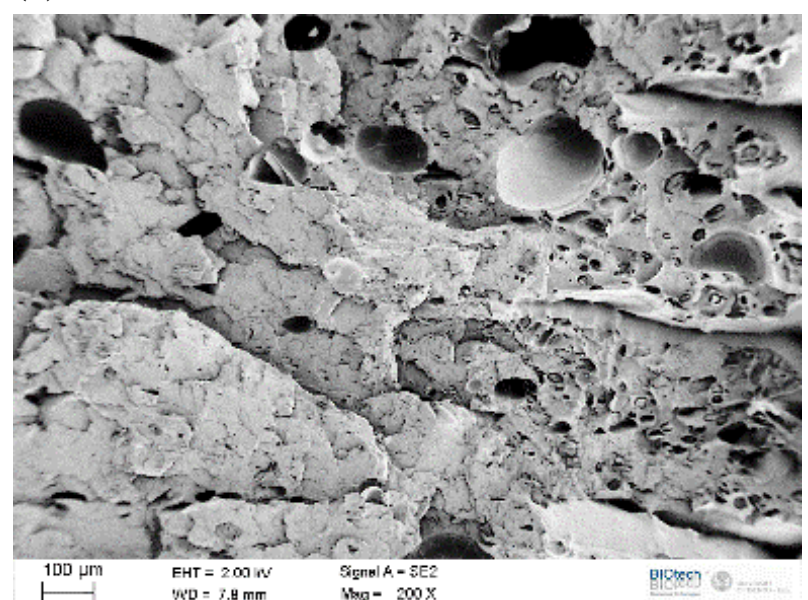

(c)

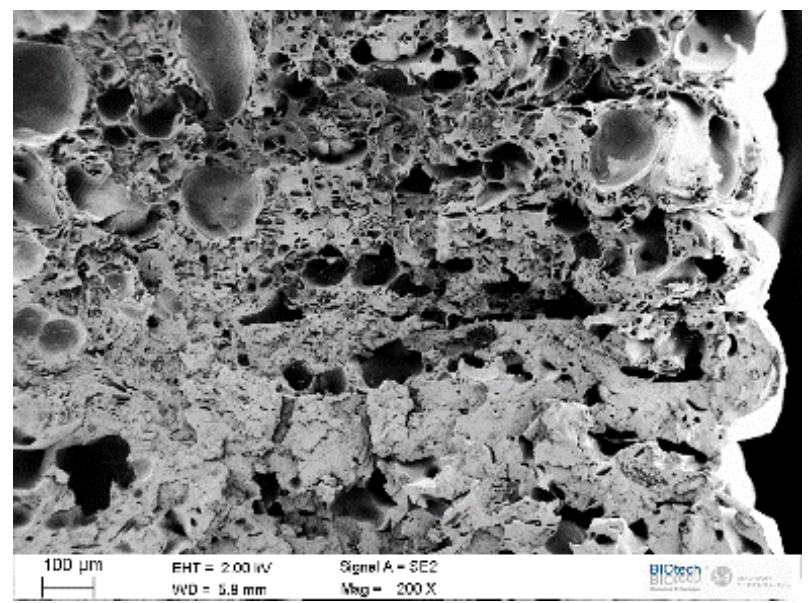

(e)

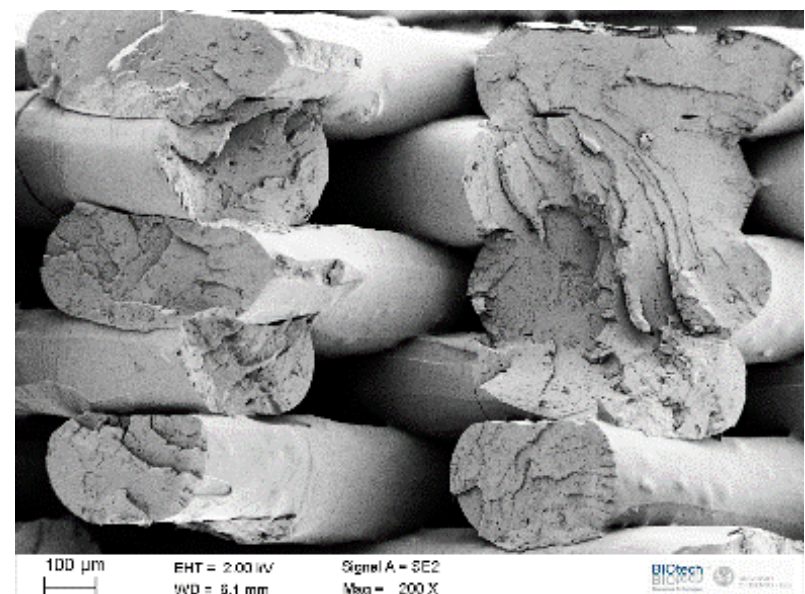

(b)

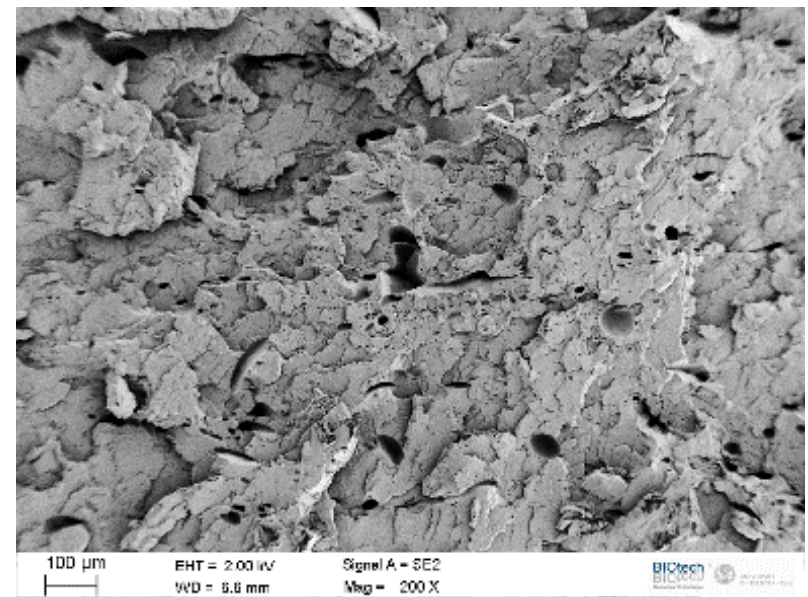

(d)

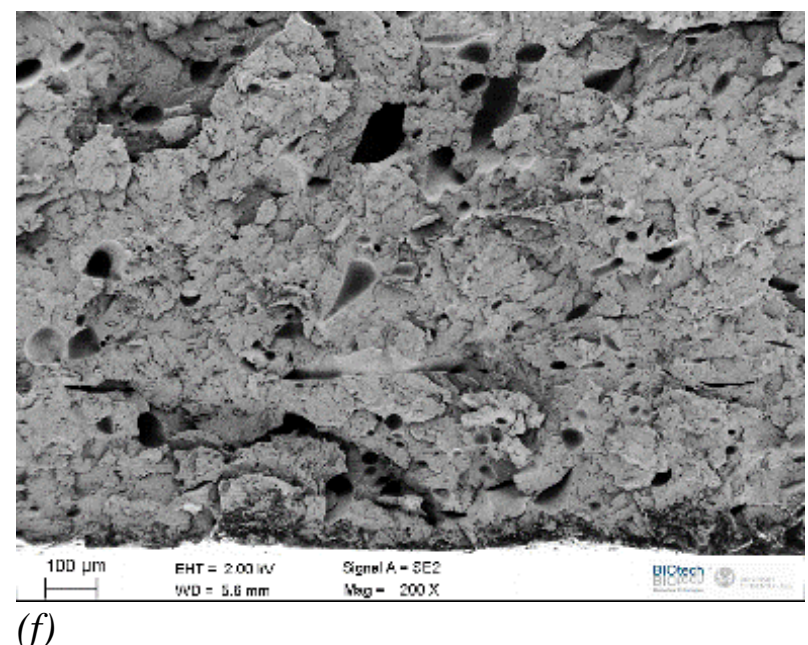

Figure 11. SEM pictures of the fracture surface after tensile test on neat PLA (a),

PLAgMAH_01MCC (b),PLA_05MCC (c),PLAgMAH_05MCC (d),PLA_10MCC $(e)$ and

PLAgMAH_1OMCC $(f)$. 
The density of 3D printed specimen was always lower compared to that of bulk material due to the porosity originated during the printing process (see Table 3).

Table 3. Density-of the filaments (bulk density) and apparent density of 3D printed specimens.

\begin{tabular}{lcc}
\hline & $\begin{array}{c}\text { Bulk density } \\
\mathrm{g} / \mathrm{cm}^{3}\end{array}$ & $\begin{array}{c}\text { Apparent density } \\
\mathrm{g} / \mathrm{cm}^{3}\end{array}$ \\
\hline PLA & $1.251 \pm 0.057$ & $0.689 \pm 0.031$ \\
PLA_01MCC & $1.241 \pm 0.028$ & $0.784 \pm 0.018$ \\
PLAgMAH_01MCC & $1.247 \pm 0.005$ & $0.717 \pm 0.003$ \\
PLA_03MCC & $1.235 \pm 0.014$ & $0.639 \pm 0.007$ \\
PLAgMAH_03MCC & $1.240 \pm 0.021$ & $0.802 \pm 0.014$ \\
PLA_05MCC & $1.221 \pm 0.030$ & $0.795 \pm 0.020$ \\
PLAgMAH_05MCC & $1.220 \pm 0.045$ & $0.822 \pm 0.030$ \\
PLA_10MCC & $1.234 \pm 0.008$ & $0.655 \pm 0.004$ \\
PLAgMAH_10MCC & $1.265 \pm 0.008$ & $0.799 \pm 0.005$ \\
\hline
\end{tabular}

The apparent density, reported in Table 3, was calculated by the ratio between the weight of the specimen and its volume (Equation 4).

$$
\text { Apparent density }=\frac{\text { weight }}{\text { volume }}
$$

The volume was approximately evaluated using the Equation 5, where $\mathrm{w}$ is the width of the specimen, $\mathrm{b}$ is the width at center $(5 \mathrm{~mm})$ of the specimen model with an area $A$ equals to $520 \mathrm{~mm}^{2}$ and $t$ is the thickness.

$$
\text { Volume }=\left(\frac{w}{b}\right)^{2} A t
$$

The ratio between apparent density and the bulk density (Eq 6), was calculated and it could be considered as an important parameter to estimate the mechanical properties of the material excluding the presence of voids and porosity.

$$
R=\frac{\text { apparent density }}{\text { bulk density }}
$$

The values of ratio $\mathrm{R}$ are reported in Figure 12. 

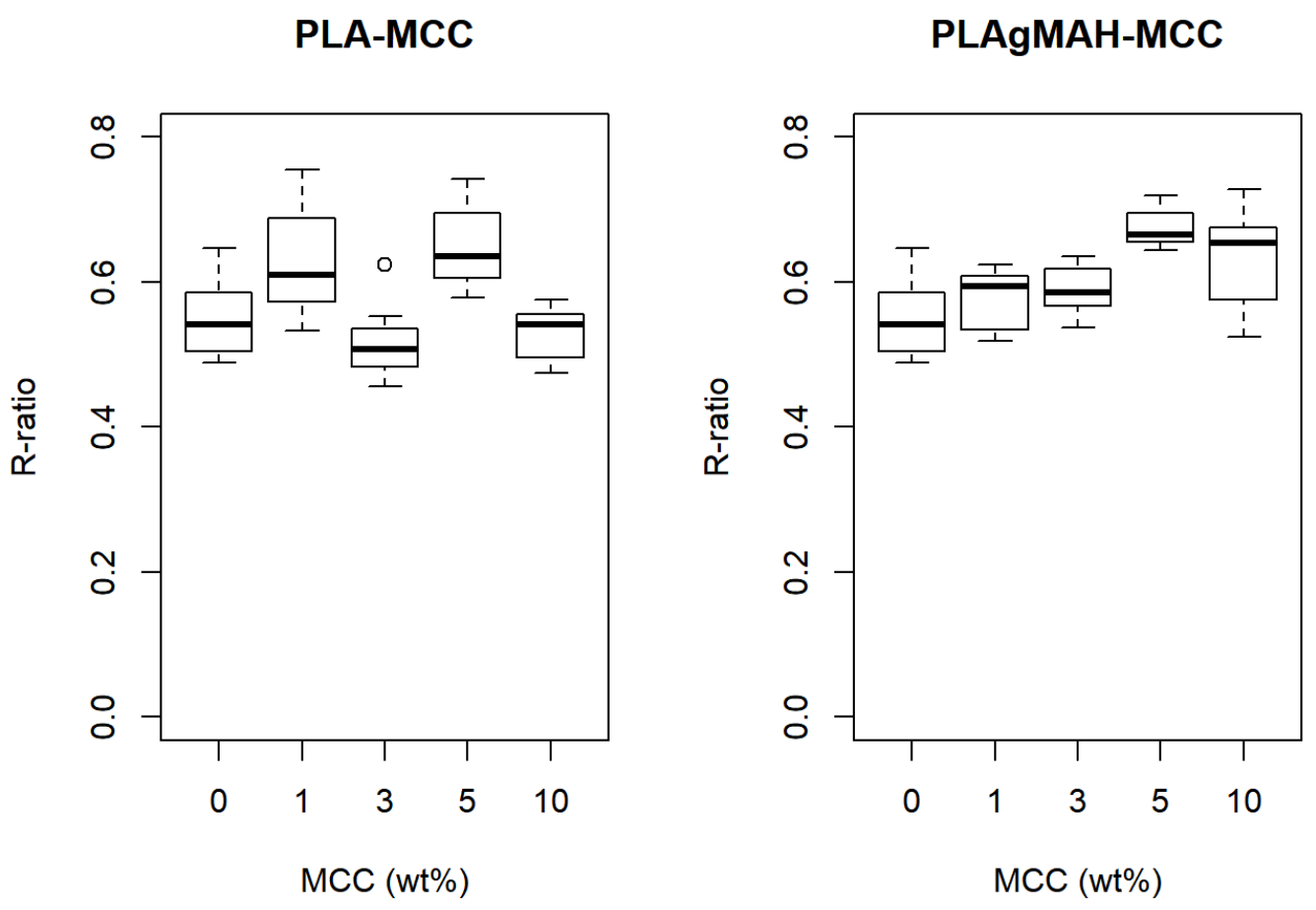

Figure 12. R-ratio as a function of wt\% of MCC for PLA and PLA grafted MAH.

ANOVA showed no interaction between $\mathrm{R}$ and $\mathrm{MCC}$, however a significative effect was found for the interaction between R and MAH (p-value = 0.034). This behavior could be explained by the pronounced decrease in viscosity due to the presence of MCC and MAH. It is important to underline that the tensile properties obtained from the mechanical tests on $3 \mathrm{D}$ printed materials reported in Figure 8 do not take into account the presence of voids within the printed structure. It is therefore clear that the properties of 3D printed specimens resulted to be lower with respect to those obtained on the filaments from which were made. A statistical analysis was performed to correlate $\mathrm{R}$ with the mechanical properties of 3D printed specimen. A significative effect was found between elastic modulus and $R(p$-value $=5.3 e-5)$ and between stress at break and $R(p$-value $=1.3 e-3)$. These results are particulary true since elastic modulus and stress at break are measured in relation of the crosssection of the specimen and so of its volume. As proof of this, ANOVA showed no interaction between strain at break and $\mathrm{R}$ in the $95 \%$ confidence interval. Maximize the $\mathrm{R}$ ratio must be a strategy to improve the stiffness and the strenght of $3 \mathrm{D}$ printed objects. 
The strength of the interface between two consecutive layers in 3D printed objects is affected by the bonding between the two layers and it is impaired by the presence of voids and defects [51]. Fracture toughness refers to a property which describes the ability of a material containing a crack to resist further fracture and it could be helpful to evaluate the interlaminar adhesion of additive manufactured objects [52, 53]. In Figure 13 is graphically illustrated the behavior of the critical stress intensity factor against the concentration of MCC in presence or not of MAH. All the samples met the requirements for a linear elastic plane strain fracture toughness measurements and therefore fracture toughness results are reported as $\mathrm{K}_{\mathrm{Q}}=\mathrm{K}_{\mathrm{IC}}$. Value found for neat 3D printed PLA, 2.25 $\pm 0.26 \mathrm{MPa}$ $\mathrm{m}^{1 / 2}$, are consistent with the ones found in literature for similar kind of materials and manufacturing technique [52, 54]. As shown in Figure 13, no significative effect was observed upon the incorporation of MCC and MAH on the fracture toughness of the 3D printed metarial along the interlaminar plane. A worsening effect was found for PLAgMAH_10MCC, p-value = 7e-4, that could be caused by the increased hardship found in printing that particular material and its brittle behavior.

PLA-MCC

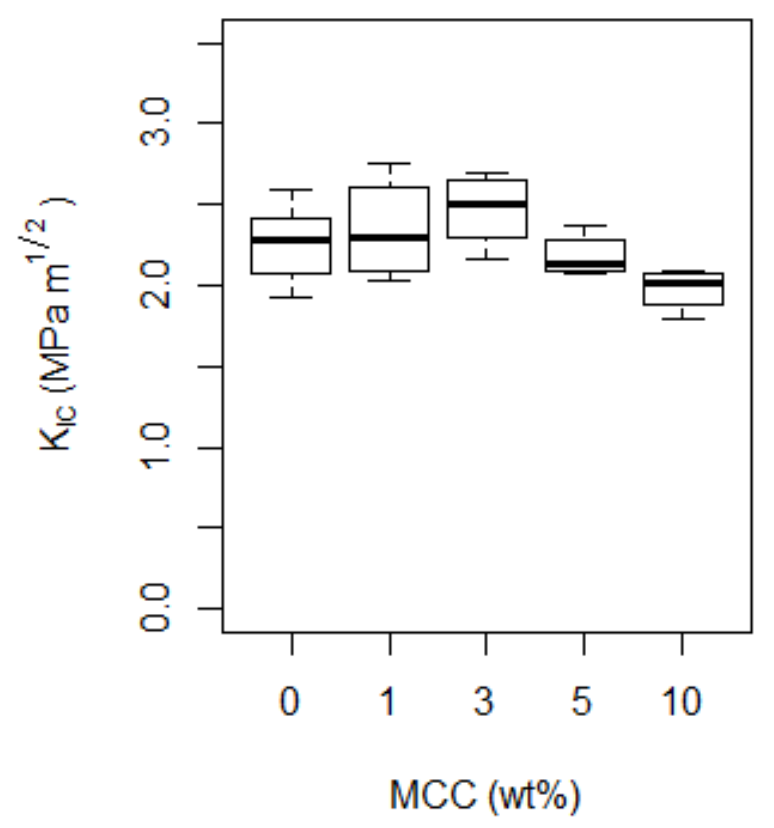

PLAgMAH-MCC

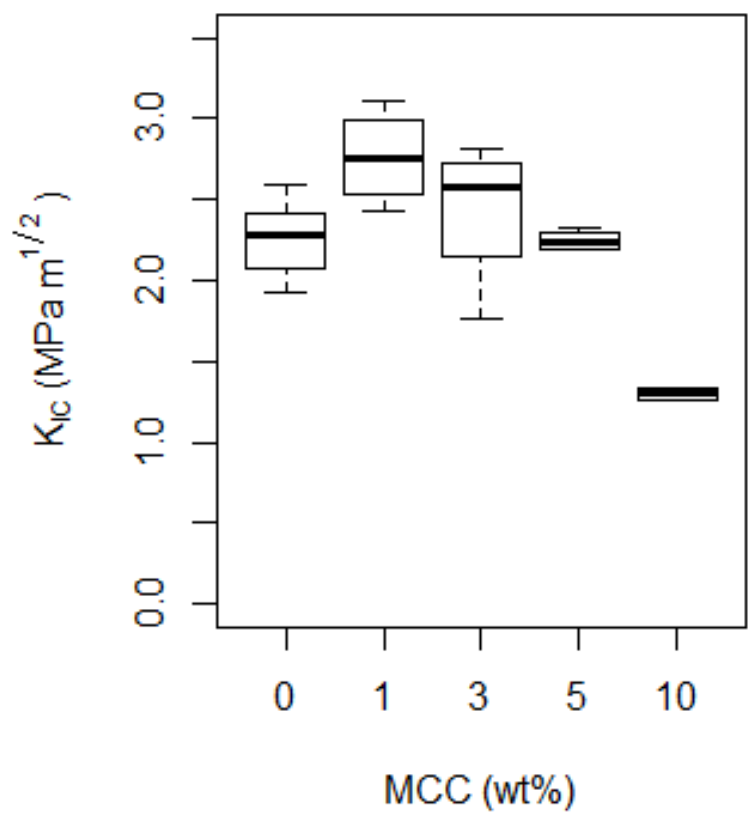

Figure 13. Critical stress intensity factor for $3 D$ printed PLA and PLAgMAH with increasing amount of $M C C$. 


\section{Conclusions}

In this work, filaments based on poly (lactic acid) (PLA) and micro crystalline cellulose (MCC) were prepared by a two-steps process (compounding and extrusion) and used to produce samples by fused filament fabrication. The effect of the addition of a compatibilizer agent was studied. In fact, maleic anhydride was grafted on PLA in a melt compounder with the aid of a peroxide. To improve the dispersion of the filler in the polymer matrix, two different types of masterbatch biocomposite were prepared at 10wt.\% of MCC, with or without MAH, i.e. PLAgMAH/MCC and PLA/MCC. Micro composite filaments were fabricated with the aid of a single screw extruder and then deposited through a 3D printer to form the final specimen. The grafting of maleic anhydride on PLA was proved through infrared spectroscopy that evidenced the presence of peaks related to the bonding of MAH. Electron microscopy was used to investigate the filler-matrix interaction and it highlighted, that upon the addition of maleic anhydride on the PLA backbone, an important improve moving from an incoherent interphase to a coherent one that suggested a good adhesion of MCC. No particular effects on the thermal degradation of PLA were revealed by thermal gravimetric analysis upon the addition of maleic anhydride and micro cellulose. According to differential scanning calorimetry analysis, micro-cellulose acted as nucleating agent for PLA shifting towards lowers temperature the crystallization peak and was observed a synergistic effect when MCC was added to PLA grafted with MAH possibly due to the increase of the chain mobility due to the low molecular weight of MAH. Stiffness of filaments was increased by the presence of micro cellulose but at the same time an embrittlement of the material was observed, this effect was amplified by the grafting with MAH. 3D printed samples demonstrated an increase in stiffness with the increasing amount of micro-cellulose and a different fracture behavior compared to bulk material due to the unique manufacturing process. The decrease in viscosity upon the addition of MCC and MAH could have enhanced the intra- and inter-layers adhesion, improving the mechanical properties at fracture of 3D printing samples. MAH was able to improve the interphase between PLA and MCC, as evidenced by SEM analysis, and therefore grafted maleic anhydride onto polylactide has been confirmed, to be a good compatibilizer to improve the adhesion of cellulose to the PLA matrix. Finally, a R-factor, defined by the ratio of apparent and bulk density, could be a useful indicator of the suitability of the investigated material for the FFF technology. It was found to be positively affected by the presence of MAH that could have improved the easiness of the printing process. Mechanical properties that depend on the cross section of specimen was also found to be related to $\mathrm{R}$ according to ANOVA. Interlayer fracture toughness was found to not be related by the presence of MCC in presence or not of MAH. Following these findings, composition at $3 \mathrm{wt} \%$ and $5 \mathrm{wt} \%$ of MCC in presence of grafted maleic anhydride could 
be suggested for the production of 3D printed specimens, as a good compromise of processing conditions, morphological structure and mechanical properties.

\section{Funding}

Not applicable

\section{Conflicts of interest/Competing interests}

Not applicable

\section{Availability of data and material}

The datasets generated during and/or analysed during the current study are available from the corresponding author on reasonable request. 


\section{References}

1. Liu, J., et al. (2019) Current advances and future perspectives of 3D printing natural-derived biopolymers. Carbohydr. Polym. 207:297-316

2. Ngo, T.D., et al. (2018) Additive manufacturing (3D printing): A review of materials, methods, applications and challenges. Composites Part B: Engineering. 143:172-196

3. Ghilan, A., et al. (2020) Trends in 3D Printing Processes for Biomedical Field: Opportunities and Challenges. J. Polym. Environ. 28(5):1345-1367

4. Singh, R., J. Singh, and S. Singh (2016) Investigation for dimensional accuracy of AMC prepared by FDM assisted investment casting using nylon- 6 waste based reinforced filament. Measurement. 78:253-259

5. Chong, S., et al. (2017) Physical Characterization and Pre-assessment of Recycled HighDensity Polyethylene as 3D Printing Material. J. Polym. Environ. 25(2):136-145

6. Dul, S., L. Fambri, and A. Pegoretti (2016) Fused deposition modelling with ABS-graphene nanocomposites. Composites Part A: Applied Science and Manufacturing. 85:181-191

7. Byberg, K.I., A.W. Gebisa, and H.G. Lemu (2018) Mechanical properties of ULTEM 9085 material processed by fused deposition modeling. Polym. Test. 72:335-347

8. Rigotti, D., A. Dorigato, and A. Pegoretti (2018) 3D printable thermoplastic polyurethane blends with thermal energy storage/release capabilities. Materials Today Communications. 15:228-235

9. Cataldi, A., et al. (2018) Polyvinyl alcohol reinforced with crystalline nanocellulose for 3D printing application. Materials Today Communications. 15:236-244

10. Chen, Z., et al. (2019) 3D printing of ceramics: A review. J. Eur. Ceram. Soc. 39(4):661-687

11. Bourell, D., et al. (2017) Materials for additive manufacturing. CIRP Annals. 66(2):659-681

12. $\mathrm{Xu}$, J., et al. (2019) Volume-forming 3D concrete printing using a variable-size square nozzle. Automation in Construction. 104:95-106

13. Liu, Z., et al. (2019) A critical review of fused deposition modeling 3D printing technology in manufacturing polylactic acid parts. The International Journal of Advanced Manufacturing Technology. 102(9-12):2877-2889

14. Pegoretti, A., L. Fambri, and C. Migliaresi (1997) In vitro degradation of poly(L-lactic acid) fibers produced by melt spinning. J Appl Polym Sci. 64(2):213-223

15. Sun, J., et al. (2018) Nanofiller Reinforced Biodegradable PLA/PHA Composites: Current Status and Future Trends. Polymers. 10(5):505

16. Swaroop, C. and M. Shukla (2018) Nano-magnesium oxide reinforced polylactic acid biofilms for food packaging applications. Int. J. Biol. Macromol. 113:729-736

17. Murariu, M. and P. Dubois (2016) PLA composites: From production to properties. Adv Drug Deliv Rev. 107:17-46

18. Oksman, K., M. Skrifvars, and J.F. Selin (2003) Natural fibres as reinforcement in polylactic acid (PLA) composites. Compos. Sci. Technol. 63(9):1317-1324

19. Mathew, A.P., K. Oksman, and M. Sain (2005) Mechanical properties of biodegradable composites from poly lactic acid (PLA) and microcrystalline cellulose (MCC). J. Appl. Polym. Sci. 97(5):2014-2025

20. Abdul Khalil, H.P.S., A.H. Bhat, and A.F. Ireana Yusra (2012) Green composites from sustainable cellulose nanofibrils: A review. Carbohydr. Polym. 87(2):963-979

21. Rzayev, Z. (2011) Graft Copolymers of Maleic Anhydride and Its Isostructural Analogues: High Performance Engineering Materials.

22. Hwang, S.W., et al. (2012) Grafting of maleic anhydride on poly(L-lactic acid). Effects on physical and mechanical properties. Polymer Testing. 31(2):333-344

23. Issaadi, K., et al. (2015) Effect of the montmorillonite intercalant and anhydride maleic grafting on polylactic acid structure and properties. Applied Clay Science. 107:62-69

24. Detyothin, S., et al. (2013) Reactive functionalization of poly(lactic acid), PLA: Effects of the reactive modifier, initiator and processing conditions on the final grafted maleic 
anhydride content and molecular weight of PLA. Polym. Degradation Stab. 98(12):26972708

25. Ma, P., et al. (2014) Melt Free-Radical Grafting of Maleic Anhydride onto Biodegradable Poly(lactic acid) by Using Styrene as A Comonomer. Polymers. 6(5):1528-1543

26. Kaynak, C. and Y. Meyva (2014) Use of maleic anhydride compatibilization to improve toughness and other properties of polylactide blended with thermoplastic elastomers. Polym. Adv. Technol. 25(12):1622-1632

27. Petersson, L., K. Oksman, and A.P. Mathew (2006) Using maleic anhydride grafted poly(lactic acid) as a compatibilizer in poly(lactic acid)/layered-silicate nanocomposites. J. Appl. Polym. Sci. 102(2):1852-1862

28. Tsou, C.-H., et al. (2014) New Composition of Maleic-Anhydride-Grafted Poly(Lactic Acid)/Rice Husk with Methylenediphenyl Diisocyanate. Mater. Sci. 20(4)

29. Zhu, R., H. Liu, and J. Zhang (2012) Compatibilizing Effects of Maleated Poly(lactic acid) (PLA) on Properties of PLA/Soy Protein Composites. Industrial \& Engineering Chemistry Research. 51(22):7786-7792

30. Nyambo, C., A.K. Mohanty, and M. Misra (2011) Effect of Maleated Compatibilizer on Performance of PLA/Wheat Straw-Based Green Composites. Macromolecular Materials and Engineering. 296(8):710-718

31. Zhang, J.F. and X. Sun (2004) Physical characterization of coupled poly(lactic acid)/starch/maleic anhydride blends plasticized by acetyl triethyl citrate. Macromol. Biosci. 4(11):1053-60

32. Siró, I. and D. Plackett (2010) Microfibrillated cellulose and new nanocomposite materials: a review. Cellulose. 17(3):459-494

33. Rigotti, D., et al. (2019) Polylactic acid-lauryl functionalized nanocellulose nanocomposites: Microstructural, thermo-mechanical and gas transport properties. Express Polymer Letters. 13(10):858-876

34. Spinella, S., et al. (2015) Polylactide/cellulose nanocrystal nanocomposites: Efficient routes for nanofiber modification and effects of nanofiber chemistry on PLA reinforcement. Polymer. 65:9-17

35. Wen, H., et al. (2019) Evaluation of Relationship Between Crystallization Structure and Thermal-Mechanical Performance of PLA with MCC Addition. ChemistrySelect. 4(34):10174-10180

36. Valentini, F., et al. (2019) Polyhydroxyalkanoates/Fibrillated Nanocellulose Composites for Additive Manufacturing. J. Polym. Environ. 27(6):1333-1341

37. Fortunati, E., et al. (2014) Investigation of thermo-mechanical, chemical and degradative properties of PLA-limonene films reinforced with cellulose nanocrystals extracted from Phormium tenax leaves. Eur. Polym. J. 56:77-91

38. Song, X., et al. (2020) Fused Deposition Modeling of Poly (lactic acid)/Nutshells Composite Filaments: Effect of Alkali Treatment. J. Polym. Environ. 28(12):3139-3152

39. Fischer, E.W., H.J. Sterzel, and G. Wegner (1973) Investigation of the structure of solution grown crystals of lactide copolymers by means of chemical reactions. Kolloid-Zeitschrift und Zeitschrift für Polymere. 251(11):980-990

40. Zhou, L., et al. (2018) Enhancing mechanical properties of poly(lactic acid) through its insitu crosslinking with maleic anhydride-modified cellulose nanocrystals from cottonseed hulls. Industrial Crops and Products. 112:449-459

41. Yu, T., N. Jiang, and Y. Li (2014) Study on short ramie fiber/poly(lactic acid) composites compatibilized by maleic anhydride. Composites Part A: Applied Science and Manufacturing. 64:139-146

42. Cooper-White, J.J. and M.E. Mackay (1999) Rheological properties of poly(lactides). Effect of molecular weight and temperature on the viscoelasticity of poly(l-lactic acid). J. Polym. Sci., Part B: Polym. Phys. 37(15):1803-1814 
43. Barczewski, M. and O. Mysiukiewicz (2018) Rheological and Processing Properties of Poly(lactic acid) Composites Filled with Ground Chestnut Shell. Polymer Korea. 42:267274

44. Brian, N.T. (2014) A review of melt extrusion additive manufacturing processes: I. Process design and modeling. Rapid Prototyping Journal. 20(3):192-204

45. Wang, T. and L.T. Drzal (2012) Cellulose-Nanofiber-Reinforced Poly(lactic acid) Composites Prepared by a Water-Based Approach. ACS Applied Materials \& Interfaces. 4(10):5079-5085

46. Khoo, R.Z., H. Ismail, and W.S. Chow (2016) Thermal and Morphological Properties of Poly (Lactic Acid)/Nanocellulose Nanocomposites. Procedia Chemistry. 19:788-794

47. Du, Y., et al. (2014) Fabrication and characterization of fully biodegradable natural fiberreinforced poly(lactic acid) composites. Composites Part B: Engineering. 56:717-723

48. Viikna, A., et al. (2012) Influence of cellulose content on thermal properties of poly(lactic) acid/cellulose and low-density polyethylene/cellulose composites. Proceedings of the Estonian Academy of Sciences. 61(3):237

49. Frone, A.N., et al. (2013) Morphology and thermal properties of PLA-cellulose nanofibers composites. Carbohydr. Polym. 91(1):377-84

50. Halász, K. and L. Csóka (2013) Plasticized Biodegradable Poly(lactic acid) Based Composites Containing Cellulose in Micro- and Nanosize. Journal of Engineering. 2013:1-9

51. Papon, E.A. and A. Haque (2019) Fracture toughness of additively manufactured carbon fiber reinforced composites. Additive Manufacturing. 26:41-52

52. Cicero, S., et al. (2021) Analysis of notch effect in the fracture behaviour of additively manufactured PLA and graphene reinforced PLA. Theor. Appl. Fract. Mech. 114:103032

53. Hart, K.R., R.M. Dunn, and E.D. Wetzel (2020) Increased fracture toughness of additively manufactured semi-crystalline thermoplastics via thermal annealing. Polymer. 211:123091

54. Chen, J., et al. (2018) Fracture Toughness Improvement of Poly(lactic acid) Reinforced with Poly $(<\mathrm{i}>\varepsilon</ \mathrm{i}>$-caprolactone $)$ and Surface-Modified Silicon Carbide. Advances in Materials Science and Engineering. 2018:6537621 